\title{
WKB-BASED SCHEMES FOR THE OSCILLATORY 1D SCHRÖDINGER EQUATION IN THE SEMI-CLASSICAL LIMIT
}

\author{
ANTON ARNOLD*, NAOUFEL BEN ABDALLAH ${ }^{\dagger}$, AND CLAUDIA NEGULESCU
}

Abstract. An efficient and accurate numerical method is presented for the solution of highly oscillatory differential equations. While standard methods would require a very fine grid to resolve the oscillations, the presented approach uses first an analytic (second order) WKB-type transformation, which filters out the dominant oscillations. The resulting ODE is much smoother and can hence be discretized on a much coarser grid, with significantly reduced numerical costs.

In many practically relevant examples, the method is even asymptotically correct w.r.t. the small parameter $\varepsilon$ that identifies the oscillation wave length. Indeed, in these cases, the error then vanishes for $\varepsilon \rightarrow 0$, even on a fixed spatial mesh. Applications to the stationary Schrödinger equation are presented.

Key words. Schrödinger equation, highly oscillating wave functions, epsilon-independent second order numerical scheme, higher order WKB-approximation, asymptotically correct finite difference scheme, asymptotic analysis

AMS subject classifications.

1. Introduction. This paper deals with an asymptotically correct scheme for the numerical solution of highly oscillating differential equations of the type

$$
\varepsilon^{2} \varphi^{\prime \prime}(x)+a(x) \varphi(x)=0,
$$

where $0<\varepsilon \ll 1$ is a very small parameter and $a(x) \geq a_{0}>0$ a sufficiently smooth function. For very small $\varepsilon>0$, the wave length $\lambda=\frac{2 \pi \varepsilon}{\sqrt{a(x)}}$ is very small, such that the solution $\varphi$ becomes highly oscillating. In a classical ODE-scheme such a situation requires a very fine mesh in order to accurately resolve the oscillations, typically at least 10 grid points per oscillation. Hence, standard numerical methods would be very costly and inefficient here. The goal of this paper is to present a new method that uses a coarse spatial grid with step size $h>\lambda$ (see Figure 1.1).

Problems that require the numerical integration of highly oscillatory equations play an essential role in a wide range of physical phenomena: electromagnetic and acoustic scattering (Maxwell and Helmholtz equations in the high frequency regime), wave evolution problems in quantum and plasma physics (Schrödinger equation in the semi-classical regime), stiff mechanical systems, and so on. Due to the high oscillations, these problems render the mathematical analysis and computation very challenging.

The application we are interested in here is the numerical solution of the stationary 1D Schrödinger equation

$$
\left\{\begin{array}{l}
-\varepsilon^{2} \psi_{E}^{\prime \prime}(x)+V(x) \psi_{E}(x)=E \psi_{E}(x), \quad x \in(0,1) \\
\psi_{E}^{\prime}(0)+i k(0) \psi_{E}(0)=0 \\
\psi_{E}^{\prime}(1)-i k(1) \psi_{E}(1)=-2 i k(1)
\end{array}\right.
$$

*Institut für Analysis und Scientific Computing, Technische Universität Wien, Wiedner Hauptstr. 8, A-1040 Wien, Austria (anton.arnold@tuwien.ac.at).

†Institut de Mathématiques de Toulouse (UMR CNRS 5219), Université de Toulouse, Université Paul Sabatier, 118, route de Narbonne, F-31062 Toulouse Cedex, France.

‡Laboratoire LATP/CMI (UMR CNRS 6632), Université de Provence, 39, rue Joliot Curie, F13453 Marseille Cedex 13, France (claudia.negulescu@cmi.univ-mrs.fr). 


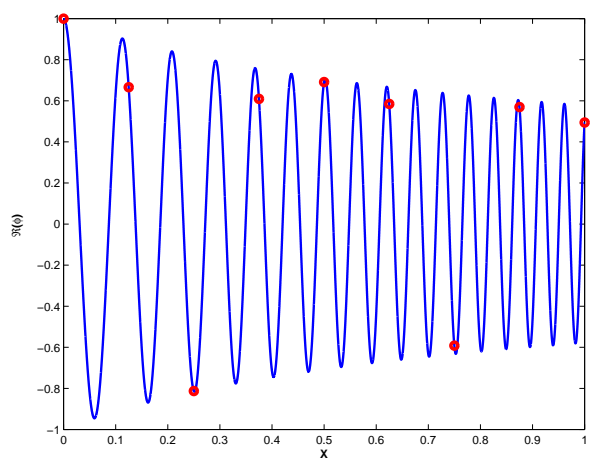

FIG. 1.1. In standard numerical methods highly oscillating solutions require a very fine mesh to capture the oscillations. However, with the analytic pre-processing of our method an accurate solution can be obtained on a coarse grid (dots). Plotted is the solution $\Re \varphi(x)$ of (1.1) with $\varepsilon=$ $0.01, h=0.125$, and $a=\left(x+\frac{1}{2}\right)^{2}$ as in $\S 3.2$.

It describes the state of an electron that is injected with the prescribed energy $E$ from the right boundary (or lead) into an electronic device (diode, e.g.), modeled on the interval $[0,1]$. The corresponding (complex valued) wave function is denoted by $\psi_{E}(x)$, where $\left|\psi_{E}(x)\right|^{2}$ is related to the spatial probability density of the electron. Due to the continuous injection of a plane wave function at $x=1$, we cannot expect $\left|\psi_{E}\right|^{2}$ to be normalized here. The small parameter $\varepsilon$ is the rescaled Planck constant and $V$ is the given electrostatic potential. We suppose that $E>V(x)$ all along the device which characterizes the problematic oscillatory regime. In contrast, the evanescent regime, i.e. $E<V(x)$, is rather harmless since the solution is then nonoscillatory, and consequently we are not considering this case in the present work. The boundary conditions in (1.2) are the so called open or transparent boundary conditions, permitting an electron wave to enter or leave the device without reflections [16]. And finally, the wave vector $k$ and the corresponding de Broglie wave-length $\lambda$ are given by

$$
k(x):=\frac{\sqrt{E-V(x)}}{\varepsilon}, \quad \lambda(x):=\frac{1}{k(x)}=\frac{\varepsilon}{\sqrt{E-V(x)}} .
$$

Solving one single ODE like (1.2) is, of course, no numerical challenge today. However, a rapid resolution of the Schrödinger equation (1.2) is a crucial element in the simulation of the electron transport in nanoscale semiconductor devices, like quantum wave-guides [1], resonant tunneling diodes (RTDs) [3], MOSFETs [20], etc. In such applications, macroscopic quantities like the electron density $n$ and the current density $j$ are given by the formulae

$$
n(x)=\int_{0}^{\infty} f(k)\left|\psi_{E(k)}(x)\right|^{2} d k, \quad j(x)=\varepsilon \int_{0}^{\infty} f(k) \Im\left(\overline{\psi_{E(k)}(x)} \psi_{E(k)}^{\prime}(x)\right) d k,
$$

where $f$ represents the injection statistics of the electrons, described by the FermiDirac or the Boltzmann distribution function. Thus, the Schrödinger equation (1.2) has to be solved many times (once per injection energy $E$ ) in order to compute the macroscopic quantities $n$ and $j$. In fact, one needs here a very fine grid in $E$, since the transition probability for the electrons through the device is very peaked w.r.t. $E$ 
(cf. [3]). Hence, efficient methods for the solution of (1.2) will lead to a considerable gain in the simulation time.

In order to put into perspective the numerical method proposed in $\S 2$, we first review the well-known WKB-approximation (cf. [15]) for the singularly perturbed ODE (1.1). The WKB-ansatz

$$
\varphi(x)=\exp \left(\frac{1}{\varepsilon} \sum_{p=0}^{\infty} \varepsilon^{p} \phi_{p}(x)\right)
$$

inserted in (1.1), leads after comparison of the $\varepsilon^{p}$-terms to

$$
\begin{aligned}
& \phi_{0}(x)= \pm i \int_{0}^{x} \sqrt{a(\tau)} d \tau+\text { const. } \\
& \phi_{1}(x)=\ln a(x)^{-1 / 4}+\text { const. } \\
& \phi_{2}(x)=\mp i \int_{0}^{x} \beta(\tau) d \tau+\text { const. }, \quad \beta:=-\frac{1}{2 a^{1 / 4}}\left(a^{-1 / 4}\right)^{\prime \prime} .
\end{aligned}
$$

The derivation of adiabatic integrators in [17] (cf. also $\S$ XIV of [5]) is closely related to using the zeroth order WKB-approximation $\varphi(x) \approx C \exp \left( \pm \frac{i}{\varepsilon} \int_{0}^{x} \sqrt{a} d \tau\right)$ to eliminate the dominant oscillations. After transformation, the resulting less oscillatory ("adiabatic") variables were approximated numerically. A localized variant of this transformation is also the background for the modified Magnus method for (1.1), developed in $\S 5$ of $[9]$.

Truncating the Ansatz (1.3) after $p=1$ yields the asymptotic approximation

$$
\varphi(x) \approx C \frac{\exp \left( \pm \frac{i}{\varepsilon} \int_{0}^{x} \sqrt{a} d \tau\right)}{\sqrt[4]{a(x)}}
$$

which is the basis to construct a WKB-finite element scheme for (1.1) in [19, 20]. While that FEM required a non-resonance condition on $h$, our new method is valid without any such restriction. Using the first order WKB-approximation (1.4) to transform (1.1) into a smoother problem was also mentioned in $\S 2.6$ of [17], but it was not pursued there numerically.

In the sequel we shall use the following notation involving the "small parameters" $\varepsilon$ and $h$ :

DEFINITION 1.1. Let $f$ be a complex valued function, and $g$ a real valued function of the two varables $\varepsilon$ and $h$. We then say that $f=\mathcal{O}_{\varepsilon, h}(g)$, iff there exists a constant $C$, such that $|f(\varepsilon, h)| \leq C g(\varepsilon, h), \forall 0<\varepsilon, h \leq 1$.

We remark that this notation shall be used not only for $\varepsilon, h$ sufficiently small, but indeed for all $0<\varepsilon, h \leq 1$. For functions of just one variable, we use the standard notation $\mathcal{O}\left(\varepsilon^{\gamma}\right)$ and $\mathcal{O}\left(h^{\gamma}\right)$ for $\varepsilon \rightarrow 0$ and $h \rightarrow 0$, resp.

Our method below is related to a second order WKB-approximation (i.e. truncation of the Ansatz (1.3) after $p=2$ ), which uses the refined asymptotics

$$
\varphi(x) \approx C \frac{\exp \left( \pm \frac{i}{\varepsilon} \phi(x)\right)}{\sqrt[4]{a(x)}}, \quad \phi(x):=\int_{0}^{x}\left(\sqrt{a(\tau)}-\varepsilon^{2} \beta(\tau)\right) d \tau .
$$

Let us briefly sketch our strategy. It is closely related to the procedure in [17], but yields a refinement to higher $\varepsilon$-order: 
1. Analytic pre-processing of (1.1) by a second order WKB-transformation of the form (1.5). The equation (1.1) is transformed into a smoother problem that can be solved accurately and efficiently on a coarse grid (see Fig. 1.1).

2. $\varepsilon$-uniform discretization of the oscillatory integral $\int \beta(y) \exp \left(\frac{2 i}{\varepsilon} \phi(y)\right) d y$ (and multiple iterates of it) appearing in the numerical scheme for the transformed, smoother problem (cf. (2.6) below).

3. Numerical integration of the phase $\phi(x)$ in (1.5). Assume that a quadrature rule (Gaussian or Chebyshev, e.g.) introduces numerical errors of the order $\mathcal{O}\left(h^{\gamma}\right)$ in the phase-computation, where $\gamma>0$ is related to the number of intermediate quadrature points (for each $h$-interval). This will typically induce $\mathcal{O}_{\varepsilon, h}\left(h^{\gamma} / \varepsilon\right)$ errors in the oscillatory integral. However, this phase integral can be computed explicitly in several relevant examples (e.g. RTDs). But even then, machine precision round-off errors will also introduce "small" $\mathcal{O}(1 / \varepsilon)$ errors.

In [17] the oscillatory integral is dealt with by first using a Taylor expansion of $\beta$ and $\phi$, followed by an integration by parts. But recently, quite refined techniques for the quadrature of oscillatory integrals were developed. The goal of the methods in [10, $18,21]$ is to provide approximations for $\int_{x}^{x+h} \beta(y) \exp \left(\frac{2 i}{\varepsilon} \phi(y)\right) d y$ of arbitrarily high $\varepsilon$-order. But for solving oscillatory ODEs there are of course additional requirements: The local discretization error must also be of sufficiently high $h$-order. Therefore let us first discuss, under this perspective, the existing techniques. The asymptotic method $[10,21]$ provides approximations of arbitrarily high $\varepsilon$-order. But since it does not yield high $h$-orders (cf. $§ 2.2$ for details), it is not usable for constructing ODE schemes. In Filon-type quadrature $[10,18]$, the oscillatory integral is approximated by integrals $\int_{x}^{x+h} \pi(y) \exp \left(\frac{2 i}{\varepsilon} \phi(y)\right) d y$, where $\pi$ is a polynomial interpolation of $\beta$, assuming that the resulting moment integrals can be evaluated exactly. Since this is not the case here, Filon-type quadrature would not apply to our situation. In the moment free Levin-type methods [21, 22], the function $\beta$ in the oscillatory integral is approximated (Hermite interpolation) by a function $L[v](y):=v^{\prime}+\frac{2 i}{\varepsilon} \phi^{\prime} v$. Then the resulting integral can be evaluated exactly. With growing interpolation order, the error has increasing orders of $\varepsilon h$. Hence, this method would appear suitable for oscillatory ODEs. But -to the authors' knowledge- it has not been used yet. In $\S 2.2$ we shall present a new variant of the asymptotic method that trades $\varepsilon$-powers into $h$-powers. The idea is to subtract from the oscillatory term $\exp \left(\frac{2 i}{\varepsilon} \phi(y)\right)$ of the integrand a trigonometric polynomial of the phase $\phi$ (which is appropriately compensated in the integration by parts). This procedure creates zeros in the integrand and increases the $h$-order of the error.

Standard methods for (1.1) (like in $[7,8]$ ) used to require a step size $h=\mathcal{O}(\varepsilon$ ) for accurate resolution. With a zeroth order WKB-transformation, the second order 2 -step scheme of [17] reduces that limitation to $h=\mathcal{O}(\sqrt{\varepsilon})$. With the choice $\gamma=4$ (Simpson rule) our second order 1-step scheme has, in general, the same $h$-limitation (see Theorem 3.1). But for explicitly integrable phases, $h$ can be chosen independently of $\varepsilon$ in our scheme.

This asymptotic correctness w.r.t. $\varepsilon$ is an additional novel feature of our scheme: RTD-models typically have piecewise linear potentials. Hence, the phase function $\phi$ in (1.5) can be integrated exactly. As a consequence, the numerical error decreases to zero as $\varepsilon \rightarrow 0$, even when using a fixed step size $h>0$. Hence, the scheme with e.g. just 2 grid points on $[0,1]$ becomes asymptotically correct in the highly oscillatory limit, which was a-priori the most difficult scenario. 
We remark that the basic "philosophy" of this asymptotic correctness is closely related to relaxation schemes or asymptotic preserving schemes. These numerical schemes are developed for PDE-families that involve a small parameter $\varepsilon$, and they stay uniformly accurate also in the scaling limit $\varepsilon \rightarrow 0$. Examples include the diffusive limits of kinetic equations [13], kinetic to fluid dynamical limits [14], and the semiclassical limit of the time dependent Schrödinger equation [4].

This paper is organized as follows. In Section 2 we present the numerical method for solving the highly oscillating equation (1.1). In $\S 3.1$ the convergence of the numerical scheme is analyzed and the main result of this paper is stated in Theorem 3.1. The advantages of the introduced methods and their $(\varepsilon, h)$-dependent error behavior are illustrated on a numerical example in $\S 3.2$. In $\S 3.3$ we add a refined error analysis to illustrate possible cancellation effects in the error accumulation for oscillatory problems. And we conclude in $\S 4$.

2. Description of the method. The objective of this paper is to solve efficiently the following highly oscillating initial value problem (IVP):

$$
\left\{\begin{array}{l}
\varepsilon^{2} \varphi^{\prime \prime}(x)+a(x) \varphi(x)=0, \quad x \in(0,1), \\
\varphi(0)=\varphi_{0}, \\
\varepsilon \varphi^{\prime}(0)=\varphi_{1} .
\end{array}\right.
$$

Here we are concerned with the scalar problem, with possibly complex valued initial conditions. The more technical vector valued situation will be addressed in a subsequent work.

To make the connection with our application presented in the introduction, we can put

$$
a(x)=E-V(x), \quad \varphi_{0}=1, \varphi_{1}=-i \sqrt{a(0)},
$$

where $E$ can be considered as fixed and $0<\varepsilon<1$ as arbitrarily small. The solution $\varphi$ of the IVP (2.1) and that one of the BVP (1.2) are then related by

$$
\psi_{E}(x)=-\frac{2 i k(1)}{\varphi^{\prime}(1)-i k(1) \varphi(1)} \varphi(x) .
$$

The existence of a solution to problem (2.1) is stated in the following proposition.

Proposition 2.1. [2, 19] Let $a \in W^{1, \infty}(0,1)$ be a given real function, satisfying $a(x) \geq a_{0}>0$ in $[0,1]$ and let $0<\varepsilon<1$ be fixed. Then the Schrödinger equation (2.1) admits a unique solution $\varphi \in W^{2, \infty}(0,1)$ verifying

$$
\|\varphi\|_{L^{\infty}(0,1)} \leq C, \quad\left\|\varepsilon \varphi^{\prime}\right\|_{L^{\infty}(0,1)} \leq C, \quad\left\|\varepsilon^{2} \varphi^{\prime \prime}\right\|_{L^{\infty}(0,1)} \leq C,
$$

with a constant $C>0$ independent of $\varepsilon$.

This predicted oscillatory behavior of $\varphi$ can be observed in Figure 2.1 (left) for two different $\varepsilon$-values.

We shall now introduce an efficient numerical method for solving (2.1). The idea is to first reformulate the oscillatory problem in order to get a new equation with "smoother" unknowns. This is the objective of Section 2.1. Then, this new problem can be solved numerically on a coarse grid (in $\S 2.2$ ). The originality of this procedure is that one fixed grid, independent of the wave-length $\varepsilon / \sqrt{a(x)}$, can be used to solve the "smooth" equation accurately, permitting thus a considerable gain in computational 
costs.

For simplicity only, we shall suppose all along this paper that $a$ is smooth enough. Note, however, that piecewise smoothness of $a$ would be enough. At discontinuities of $a$, the IVP could just be "restarted".

Hypothesis A Let $a \in C^{\infty}[0,1]$ be a fixed smooth (real valued) function, satisfying $a(x) \geq a_{0}>0$ in $[0,1]$, which means that we are in the oscillatory regime. Besides, let $0<\varepsilon<1$ be an arbitrary real number.

2.1. Reformulation of the continuous problem. The first step in our reformulation is to pass from the second order differential equation (2.1) to a system of first order differential equations. Motivated by the form of (1.5) we set

$$
U(x)=\left(\begin{array}{c}
u_{1} \\
u_{2}
\end{array}\right):=\left(\begin{array}{c}
a^{1 / 4} \varphi(x) \\
\frac{\varepsilon\left(a^{1 / 4} \varphi\right)^{\prime}(x)}{\sqrt{a(x)}}
\end{array}\right),
$$

we have

$$
\left\{\begin{array}{l}
U^{\prime}(x)=\left[\frac{1}{\varepsilon} A_{0}(x)+\varepsilon A_{1}(x)\right] U(x), \quad 0<x<1 \\
U(0)=U_{I}
\end{array}\right.
$$

with the two matrices

$$
A_{0}(x)=\sqrt{a(x)}\left(\begin{array}{cc}
0 & 1 \\
-1 & 0
\end{array}\right) ; \quad A_{1}(x)=\left(\begin{array}{cc}
0 & 0 \\
2 \beta(x) & 0
\end{array}\right)
$$

where

$$
\beta=-\frac{1}{2 a^{1 / 4}}\left(a^{-1 / 4}\right)^{\prime \prime}
$$

Note that in (2.2) the matrix $\frac{1}{\varepsilon} A_{0}=\mathcal{O}\left(\varepsilon^{-1}\right)$ is dominant and gives rise to the highly oscillatory part in the solution. On the other hand, $\varepsilon A_{1}$ is a small $\mathcal{O}(\varepsilon)$-perturbation. In contrast, [17] uses $\tilde{U}(x):=\left(\varphi(x), \varepsilon \varphi^{\prime}(x) / \sqrt{a(x)}\right)^{\top}$ to transform (2.1) to a first order system. And this results in an $\mathcal{O}(1)$-perturbation of the dominant matrix $\frac{1}{\varepsilon} A_{0}(x)$.

Proposition 2.1 implies the existence of a unique solution $U \in\left(W^{1, \infty}(0,1)\right)^{2}$ of (2.2), satisfying

$$
\|U\|_{L^{\infty}(0,1)} \leq C \quad ; \quad\left\|U^{\prime}\right\|_{L^{\infty}(0,1)} \leq \frac{C}{\varepsilon},
$$

with a constant $C>0$ independent of $\varepsilon$.

As a second step we diagonalize now the dominant part of the system (2.2) by performing the change of variable

$$
Y(x):=P U(x),
$$

where

$$
P:=\frac{1}{\sqrt{2}}\left(\begin{array}{cc}
i & 1 \\
1 & i
\end{array}\right) \quad ; \quad P^{-1}=\frac{1}{\sqrt{2}}\left(\begin{array}{cc}
-i & 1 \\
1 & -i
\end{array}\right)
$$



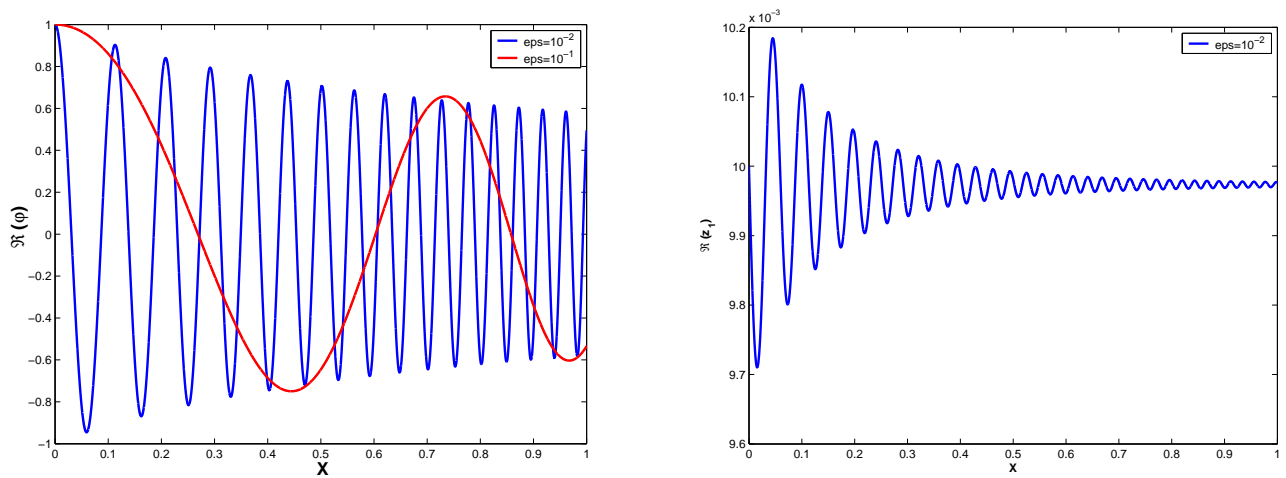

FIG. 2.1. Left: solution $\Re \varphi(x)$ of (2.1) for 2 values of $\varepsilon$. Right: solution $\Re z_{1}(x)$ of (2.6). The simulation is performed for the same potential $a=(x+1 / 2)^{2}$ as in §3.2.

The equation for $Y$ reads

$$
\left\{\begin{array}{l}
\frac{d Y}{d x}=\frac{i}{\varepsilon} D^{\varepsilon} Y+\varepsilon N Y, \quad 0<x<1 \\
Y(0)=Y_{I}
\end{array}\right.
$$

with

$$
D^{\varepsilon}(x)=\left(\begin{array}{cc}
D_{1}^{\varepsilon}(x) & 0 \\
0 & D_{2}^{\varepsilon}(x)
\end{array}\right)=\left(\begin{array}{cc}
\sqrt{a}-\varepsilon^{2} \beta & 0 \\
0 & -\sqrt{a}+\varepsilon^{2} \beta
\end{array}\right) ; N(x)=\beta(x)\left(\begin{array}{cc}
0 & 1 \\
1 & 0
\end{array}\right) .
$$

Again, the matrix $\frac{i}{\varepsilon} D^{\varepsilon}$ gives rise to highly oscillatory solutions, while $\varepsilon N$ is a small perturbation.

In our final transformation step we eliminate the leading oscillations by defining the diagonal matrix

$$
\Phi^{\varepsilon}(x):=\int_{0}^{x} D^{\varepsilon}(y) d y=\left(\begin{array}{cc}
\phi^{\varepsilon} & 0 \\
0 & -\phi^{\varepsilon}
\end{array}\right),
$$

with the (real valued) phase function

$$
\phi^{\varepsilon}(x):=\int_{0}^{x}\left(\sqrt{a(\tau)}-\varepsilon^{2} \beta(\tau)\right) d \tau .
$$

Note that this is precisely the phase in the second order WKB-approximation (1.5). Making the change of unknown

$$
Z(x)=\left(\begin{array}{c}
z_{1} \\
z_{2}
\end{array}\right):=\exp \left(-\frac{i}{\varepsilon} \Phi^{\varepsilon}(x)\right) Y(x),
$$

leads to the system

$$
\left\{\begin{array}{l}
\frac{d Z}{d x}=\varepsilon N^{\varepsilon} Z, \quad 0<x<1 \\
Z(0)=Z_{I}=Y_{I}
\end{array}\right.
$$

Here, the matrix

$$
N^{\varepsilon}=\exp \left(-\frac{i}{\varepsilon} \Phi^{\varepsilon}\right) N \exp \left(\frac{i}{\varepsilon} \Phi^{\varepsilon}\right)
$$


is bounded independently of $\varepsilon$. It is off-diagonal, with the entries

$$
N_{1,2}^{\varepsilon}(x)=\beta(x) e^{-\frac{2 i}{\varepsilon} \phi^{\varepsilon}(x)}, \quad N_{2,1}^{\varepsilon}(x)=\beta(x) e^{\frac{2 i}{\varepsilon} \phi^{\varepsilon}(x)} .
$$

Note that, due to our higher order WKB-transformation, the ODE (2.6) has a trivial limit (for $\varepsilon \rightarrow 0$ ): Its solution converges to $Z^{0}(x):=Z_{I}$ (uniformly in $x$ ). This limit is trivial to capture numerically and the core of the above mentioned asymptotic correctness (w.r.t. $\varepsilon$ ) of the presented scheme. The importance of recovering numerically such adiabatic invariants of (2.1) was already mentioned in $\S 3$ of [9].

Let us briefly compare this asymptotic behavior of (2.6) to related approaches in the literature: In $\S 2.5$ of [17], e.g., the r.h.s. of the corresponding equation was only $\mathcal{O}(1)$ as $\varepsilon \rightarrow 0$, which rendered their final scheme $\varepsilon$-uniformly convergent (but not asymptotically correct). However, when basing their scheme on the adiabatically invariant variables (as mentioned in $\S 2.6$ of [17]), it would also be asymptotically correct.

We remark that the concept of super-adiabatic transformations (cf. §XIV.1 in [5]) has the same goal, i.e. transforming a first order ODE such that the new system matrix is of the order $\mathcal{O}\left(\varepsilon^{k}\right)$ for some $k \in \mathbb{N}$. Actually, §XIV.1 is presented for linear ODE systems with a skew-Hermitian system matrix (which is not the case in (2.2), e.g.). However, it can easily be extended to diagonalizable system matrices. When this extended method is applied to $(2.1)$ with $\widetilde{U}(x):=\left(\varphi(x), \varepsilon \varphi^{\prime}(x)\right)^{\top}$, it yields a transformed system again of form (2.6). However, the resulting system matrix then is of a much more complex and lenghty form than our formulation (2.6).

The principal idea of this paper is that, instead of solving (2.2) (or (2.1)), we shall solve numerically (2.6) and recover then the original solution by

$$
U(x)=P^{-1} e^{\frac{i}{\varepsilon} \Phi^{\varepsilon}(x)} Z(x) .
$$

In particular this inverse transform of the asymptotic limit $Z_{I}=\left(z_{1}, z_{2}\right)^{\top}$ is given by

$$
\varphi(x)=\frac{1}{\sqrt{2} \sqrt[4]{a(x)}}\left(-i e^{\frac{i}{\varepsilon} \phi^{\varepsilon}(x)} z_{1}+e^{-\frac{i}{\varepsilon} \phi^{\varepsilon}(x)} z_{2}\right),
$$

which is a linear combination of the two second order WKB-functions (1.5).

Let us explain in some words the essential advantage of doing so. For small $\varepsilon$, the function $U$ is highly oscillating, with amplitude of order $\mathcal{O}(1)$ as $\varepsilon \rightarrow 0$ (see (2.3) and Figure 2.1). Thus, a very fine grid (e.g. 10 discretization points per wave length) has to be chosen in order to solve accurately enough the system (2.2). The function $Z$ is also highly oscillating - approximately with the same frequency as $U$. But, as a great advantage, $Z$ oscillates around a smooth function, with an amplitude only of the order $\mathcal{O}\left(\varepsilon^{2}\right)$ (see (2.10) and Figure 2.1). Hence, if we take a coarse grid with a step size $h \gg \varepsilon$ (step size superior to the wave-length), then we shall incur at maximum an error of the order $\mathcal{O}\left(\varepsilon^{2}\right)$ when solving (2.6), whereas for (2.2) the error would be of the order $\mathcal{O}(1)$ as $\varepsilon \rightarrow 0$. This is summarized in the following proposition.

Proposition 2.2. Under Hypothesis A, the problem (2.6) admits a unique solution $Z \in C^{\infty}[0,1]$ with the explicit form

$$
Z(x)=Z_{I}+\sum_{p=1}^{\infty} \varepsilon^{p} M_{p}^{\varepsilon}(x ; 0) Z_{I},
$$


where the matrices $M_{p}^{\varepsilon}, p \geq 1$ are given by

$$
\begin{aligned}
& M_{p}^{\varepsilon}(\eta ; \xi)=\int_{\xi}^{\eta} \int_{\xi}^{y_{1}} \cdots \int_{\xi}^{y_{p-1}} N^{\varepsilon}\left(y_{1}\right) \cdots N^{\varepsilon}\left(y_{p}\right) d y_{p} \cdots d y_{1}, \\
& M_{p}^{\varepsilon}(\eta ; \xi)=\int_{\xi}^{\eta} N^{\varepsilon}(y) M_{p-1}^{\varepsilon}(y ; \xi) d y, \quad M_{0}^{\varepsilon}=I d .
\end{aligned}
$$

Moreover we have the estimates

$$
\left\|Z-Z_{I}\right\|_{L^{\infty}(0,1)} \leq C \varepsilon^{2}, \quad\left\|Z^{\prime}\right\|_{L^{\infty}(0,1)} \leq C \varepsilon, \quad\left\|Z^{\prime \prime}\right\|_{L^{\infty}(0,1)} \leq C,
$$

with a constant $C>0$ independent of $\varepsilon$.

Proof. Integrating the equation for $Z,(2.6)$, over the interval $(\xi, \eta)$ we find

$$
\begin{aligned}
Z(\eta) & =Z(\xi)+\varepsilon \int_{\xi}^{\eta} N^{\varepsilon}\left(y_{1}\right) Z\left(y_{1}\right) d y_{1} \\
& =Z(\xi)+\varepsilon\left(\int_{\xi}^{\eta} N^{\varepsilon}\left(y_{1}\right) d y_{1}\right) Z(\xi)+\varepsilon^{2} \int_{\xi}^{\eta} N^{\varepsilon}\left(y_{1}\right) \int_{\xi}^{y_{1}} N^{\varepsilon}\left(y_{2}\right) Z\left(y_{2}\right) d y_{2} d y_{1} \\
& =Z(\xi)+\sum_{p=1}^{\infty} \varepsilon^{p} M_{p}^{\varepsilon}(\eta ; \xi) Z(\xi),
\end{aligned}
$$

with $M_{p}^{\varepsilon}$ defined in (2.9). In order to show the convergence of this series, as well as the estimate (2.10), we shall estimate the terms $M_{p}^{\varepsilon}$. Let us start with $M_{1}^{\varepsilon}$ and the two representations:

$$
\begin{aligned}
m_{1}^{\varepsilon}(\eta ; \xi) & :=\left(M_{1}^{\varepsilon}(\eta ; \xi)\right)_{2,1}=\overline{\left(M_{1}^{\varepsilon}(\eta ; \xi)\right)_{1,2}} \\
2.12) & =\int_{\xi}^{\eta} \beta(y) e^{\frac{2 i}{\varepsilon} \phi^{\varepsilon}(y)} d y=-\left.i \varepsilon \beta_{0}(y) e^{\frac{2 i}{\varepsilon} \phi^{\varepsilon}(y)}\right|_{\xi} ^{\eta}+i \varepsilon \int_{\xi}^{\eta} \beta_{0}^{\prime}(y) e^{\frac{2 i}{\varepsilon} \phi^{\varepsilon}(y)} d y,
\end{aligned}
$$

where

$$
\beta_{0}(y):=\frac{\beta}{2 \phi^{\prime}}(y)=\frac{\beta}{2\left(\sqrt{a}-\varepsilon^{2} \beta\right)}(y) .
$$

Note that (2.5) and Hypothesis A imply the following estimates (uniform in $0<\varepsilon \leq \varepsilon_{0}$ for some $\varepsilon_{0}$ sufficiently small)

$$
\|\beta\|_{L^{\infty}(0,1)} \leq B ; \quad\left\|\beta_{0}\right\|_{L^{\infty}(0,1)} \leq B_{0} ; \quad\left\|\beta_{0}^{\prime}\right\|_{L^{\infty}(0,1)} \leq B_{1} .
$$

Then (2.12) implies

$$
\left\|M_{1}^{\varepsilon}(\eta ; \xi)\right\|_{\infty} \leq \min \left(B|\eta-\xi|, \varepsilon\left[2 B_{0}+B_{1}|\eta-\xi|\right]\right),
$$

where $\|.\|_{\infty}$ denotes the $\infty$-matrix norm in $\mathbb{C}^{2 \times 2}$. Note that the $\varepsilon$-order in the second term is due to $N^{\varepsilon}$ being oscillating at the frequency $\frac{1}{\varepsilon}$. From (2.9) we then obtain the estimate for each $p \geq 1$ :

$$
\left\|M_{p}^{\varepsilon}(\eta ; \xi)\right\|_{\infty} \leq B^{p-1} \frac{|\eta-\xi|^{p-1}}{p !} \min \left(B|\eta-\xi|, \varepsilon\left[2 p B_{0}+B_{1}|\eta-\xi|\right]\right), \quad p \geq 1 .
$$

This shows the convergence of (2.8). Also, the terms involved in the expansion of $Z(x)-Z_{I}$ are at least of the order $\varepsilon^{2}$ which implies the estimates (2.10). 
Corollary 2.3. The matrices $M_{p}^{\varepsilon}(\eta ; \xi)$ involved in the series (2.8) can be estimated as follows:

$$
\left\|M_{p}^{\varepsilon}(\eta ; \xi)\right\|_{\infty} \leq B^{p-1} \frac{|\eta-\xi|^{p-1}}{p !} \min \left(B|\eta-\xi|, \varepsilon\left[2 p B_{0}+B_{1}|\eta-\xi|\right]\right), p \geq 1,
$$

with $B, B_{0}, B_{1}$ positive constants given in (2.13). Hence, we have for fixed $p \geq 1$

$$
M_{p}^{\varepsilon}(\xi+h ; \xi)=\mathcal{O}_{\varepsilon, h}\left(h^{p-1} \min (\varepsilon, h)\right) .
$$

To summarize, the solution $U$ of the initial problem (2.2) is highly oscillating for $\varepsilon \ll 1$. Standard methods would therefore require to use very fine meshes. Here, we will solve instead the reformulated problem (2.6) and transform back to the original variable $U$ via (2.7). As will be seen in Section 3, this procedure allows to use coarser grids $(h>\varepsilon)$, leading to a considerably gain in simulation time.

2.2. Numerical discretization of the transformed problem. The aim of this section is to introduce a first order and a second order scheme to solve the IVP

$$
\left\{\begin{array}{l}
\frac{d Z}{d x}=\varepsilon N^{\varepsilon} Z, \quad 0<x<1 \\
Z(0)=Z_{I}
\end{array}\right.
$$

Since $N^{\varepsilon}$ is highly oscillatory, we shall pay attention to the $\varepsilon$-uniform discretization of the oscillatory integrals. Let $0=x_{1}<\cdots<x_{n}<\cdots<x_{N}=1$ be a discretization of the interval $(0,1)$ and $h:=\max _{n=1, \cdots, N-1}\left|x_{n+1}-x_{n}\right|$. In the following, for simplicity, we shall often denote the cell $I_{n}:=\left(x_{n}, x_{n+1}\right)$ simply by $(\xi, \eta)$, i.e. $\xi=x_{n}$ and $\eta=x_{n+1}$.

In order to design a scheme of order $P$ in the step size $h$, we shall start from the formula (2.8) (limit of Picard iteration)

$$
Z(\eta)=Z(\xi)+\sum_{p=1}^{\infty} \varepsilon^{p} M_{p}^{\varepsilon}(\eta ; \xi) Z(\xi)
$$

and proceed in two steps.

Step 1: First we truncate the series (2.16) at order $P$, the remainder being of the order $\varepsilon^{P+1} h^{P} \min (\varepsilon, h)$. Indeed, due to $(2.14)$

$$
\left\|\sum_{p=P+1}^{\infty} \varepsilon^{p} M_{p}^{\varepsilon}(\eta ; \xi)\right\|_{\infty} \leq C \frac{B^{P}}{(P+1) !} \varepsilon^{P+1} h^{P} \min (h B, \varepsilon(P+1)) .
$$

For each fixed $\varepsilon$ this will lead to a scheme of order $P$ w.r.t. the step size $h$.

Step 2: Since the highly oscillating integrals $M_{p}^{\varepsilon}$ cannot be computed exactly, we shall make another approximation. Here, it is our goal to incur an additional discretization error of the same order as in Step 1 (which is not always possible). Anyhow, the error will decay as $\varepsilon \rightarrow 0$. As usual for oscillatory integrals, our approximation will rely on integrations by parts. But since we are dealing here with two small parameters, two different expansions of $M_{p}^{\varepsilon}$ are possible: The expansion w.r.t. $h$ permits to increase the error order in $h$, and the $\varepsilon$-expansion the error order in $\varepsilon$. But the error term from Step 1 has a mixed order in $h$ and $\varepsilon$. Thus, we need to combine the two mentioned expansions. In the sequel we present this procedure for $M_{1}^{\varepsilon}$ and $M_{2}^{\varepsilon}$. 
$h$-expansion of $M_{1}^{\varepsilon}$. Using the notation in the proof of Prop. 2.2 we compute

$$
\begin{gathered}
m_{1}^{\varepsilon}(\eta ; \xi)=\left(M_{1}^{\varepsilon}(\eta ; \xi)\right)_{2,1}=\int_{\xi}^{\eta} \beta(y) e^{\frac{2 i}{\varepsilon} \phi(y)} d y=e^{\frac{2 i}{\varepsilon} \phi(\xi)} \int_{\xi}^{\eta} \beta(y) e^{\frac{2 i}{\varepsilon}(\phi(y)-\phi(\xi))} d y \\
=-(i \varepsilon) e^{\frac{2 i}{\varepsilon} \phi(\xi)} \int_{\xi}^{\eta} \frac{\beta}{2 \phi^{\prime}}(y) \frac{d}{d y}\left(e^{\frac{2 i}{\varepsilon}(\phi(y)-\phi(\xi))}-1\right) d y \\
=-(i \varepsilon) e^{\frac{2 i}{\varepsilon} \phi(\xi)}\left\{\left[\beta_{0}(y) H_{1}\left(\frac{2}{\varepsilon}(\phi(y)-\phi(\xi))\right)\right]_{\xi}^{\eta}-\int_{\xi}^{\eta} \beta_{0}^{\prime}(y) H_{1}\left(\frac{2}{\varepsilon}(\phi(y)-\phi(\xi))\right) d y\right\} \\
=-(i \varepsilon) e^{\frac{2 i}{\varepsilon} \phi(\xi)}\left\{\beta_{0}(\eta) H_{1}\left(\frac{2}{\varepsilon}(\phi(\eta)-\phi(\xi))\right)+i \varepsilon \int_{\xi}^{\eta} \beta_{1}(y) H_{2}\left(\frac{2}{\varepsilon}(\phi(y)-\phi(\xi))\right)^{\prime} d y\right\} \\
=-(i \varepsilon) e^{\frac{2 i}{\varepsilon} \phi(\xi)}\left\{\beta_{0}(\eta) H_{1}\left(\frac{2}{\varepsilon}(\phi(\eta)-\phi(\xi))\right)+i \varepsilon \beta_{1}(\eta) H_{2}\left(\frac{2}{\varepsilon}(\phi(\eta)-\phi(\xi))\right)\right\} \\
+\mathcal{O}_{\varepsilon, h}\left(h^{2} \min (\varepsilon, h)\right),
\end{gathered}
$$

where

$$
\begin{gathered}
H_{k}(\eta):=e^{i \eta}-\sum_{p=0}^{k-1} \frac{(i \eta)^{p}}{p !} \\
\beta_{0}(y):=\frac{\beta}{2 \phi^{\prime}}(y)=\frac{\beta}{2\left(\sqrt{a}-\varepsilon^{2} \beta\right)}(y) ; \quad \beta_{k+1}:=\frac{1}{2 \phi^{\prime}(y)} \frac{d}{d y}\left(\beta_{k}\right)(y) .
\end{gathered}
$$

The key idea in $(2.18)$ is to shift the oscillatory factor $\exp \left(\frac{2 i}{\varepsilon} \phi(y)\right)$ by -1 to create a zero in $[\xi, \eta]$. And this increases the $h$-order of the remainder in each integration by parts. Continuing iteratively we obtain the asymptotic expansion (w.r.t. $h$ )

$$
m_{1}^{\varepsilon}(\eta ; \xi) \sim-\sum_{k=1}^{\infty}(i \varepsilon)^{k} e^{\frac{2 i}{\varepsilon} \phi(\xi)} \beta_{k-1}(\eta) H_{k}\left(\frac{2}{\varepsilon}(\phi(\eta)-\phi(\xi))\right)
$$

But just as in $\S 3.1$ of [10], the series (2.19) might not converge.

Note that (2.5) and Hypothesis A imply $\phi^{\prime}(x) \geq \phi_{0}>0$ for $\varepsilon$ sufficiently small. Hence, the phase $\phi$ has no stationary point and $\left\|\beta_{k}\right\|_{L^{\infty}(0,1)}$ is uniformly bounded w.r.t. small $\varepsilon$. Since

$$
H_{k}(\eta)=\mathcal{O}\left(\min \left(\eta^{k}, \eta^{k-1}\right)\right),
$$

the $k^{t h}$ term of the expansion of $m_{1}^{\varepsilon}$ is of the order $h^{k-1} \min (\varepsilon, h)$ (due to the $k$-th order zero of $H_{k}$ at $\left.\eta=0\right)$. Keeping only the first term yields an error for $m_{1}^{\varepsilon}(\eta ; \xi)$ of order $h \min (\varepsilon, h)$, thus producing an order 1 scheme (uniformly in $\varepsilon$ ). Keeping the first two terms yields an error of order $h^{2} \min (\varepsilon, h)$, thus producing an order 2 scheme (uniformly in $\varepsilon$ ).

$\varepsilon-$ expansion of $M_{1}^{\varepsilon}$. The asymptotic method for oscillatory integrals (cf. $\S 2$ of 
[10], $\S 2$ of [21]) yields a slightly different expansion of $m_{1}^{\varepsilon}$ :

$$
\begin{aligned}
m_{1}^{\varepsilon}(\eta ; \xi) & =-(i \varepsilon)\left[\beta_{0}(y) e^{\frac{2 i}{\varepsilon} \phi(y)}\right]_{\xi}^{\eta}-(i \varepsilon)^{2}\left[\beta_{1}(y) e^{\frac{2 i}{\varepsilon} \phi(y)}\right]_{\xi}^{\eta}+(i \varepsilon)^{2} \int_{\xi}^{\eta} \beta_{1}^{\prime}(y) e^{\frac{2 i}{\varepsilon} \phi(y)} d y \\
& \sim-\sum_{k=1}^{\infty}(i \varepsilon)^{k}\left[\beta_{k-1}(\eta) e^{\frac{2 i}{\varepsilon} \phi(\eta)}-\beta_{k-1}(\xi) e^{\frac{2 i}{\varepsilon} \phi(\xi)}\right]
\end{aligned}
$$

where the $k^{t h}$ term in this expansion is of the order $\varepsilon^{k-1} \min (\varepsilon, h)$. In contrast to (2.18), this expansion yields higher $\varepsilon$-orders with each integration by parts.

Mixed $\varepsilon-h-$ expansion of $M_{1}^{\varepsilon}$. In order to design a first order scheme for (2.15), we recall from Step 1 (with $P=1$ ) that

$$
Z\left(x_{n+1}\right)=Z\left(x_{n}\right)+\varepsilon M_{1}^{\varepsilon}\left(x_{n+1} ; x_{n}\right) Z\left(x_{n}\right)+\gamma_{n}, \quad \text { with } \quad \gamma_{n}=\mathcal{O}_{\varepsilon, h}\left(\varepsilon^{2} h \min (\varepsilon, h)\right)
$$

The idea is now to derive a mixed expansion of $M_{1}^{\varepsilon}\left(x_{n+1} ; x_{n}\right)$ that reduces the consistency error to the same magnitude as $\gamma_{n}$ (i.e. $\mathcal{O}_{\varepsilon, h}(\varepsilon h \min (\varepsilon, h))$ for $\left.m_{1}^{\varepsilon}(\eta ; \xi)\right)$. To this end we shall first make one step of the standard asymptotic method (2.21). This increases the $\varepsilon$-power of the error to $\mathcal{O}_{\varepsilon, h}(\varepsilon \min (\varepsilon, h))$ :

$$
\begin{aligned}
m_{1}^{\varepsilon}(\eta ; \xi) & =\int_{\xi}^{\eta} \beta(y) e^{\frac{2 i}{\varepsilon} \phi(y)} d y=-i \varepsilon \int_{\xi}^{\eta} \beta_{0}(y)\left[e^{\frac{2 i}{\varepsilon} \phi(y)}\right]^{\prime} d y \\
& =-i \varepsilon\left[\beta_{0}(y) e^{\frac{2 i}{\varepsilon} \phi(y)}\right]_{\xi}^{\eta}+i \varepsilon \int_{\xi}^{\eta} \beta_{0}^{\prime}(y) e^{\frac{2 i}{\varepsilon} \phi(y)} d y \\
& =-i \varepsilon\left[\beta_{0}(y) e^{\frac{2 i}{\varepsilon} \phi(y)}\right]_{\xi}^{\eta}+T_{1}^{\varepsilon}(\eta, \xi) .
\end{aligned}
$$

Next we make one step of the "shifted asymptotic method" (2.18). This increases the order of the method w.r.t. $h$. Indeed,

$$
\begin{aligned}
T_{1}^{\varepsilon}(\eta, \xi) & =i \varepsilon \int_{\xi}^{\eta} \beta_{0}^{\prime}(y) e^{\frac{2 i}{\varepsilon} \phi(y)} d y=-(i \varepsilon)^{2} e^{\frac{2 i}{\varepsilon} \phi(\xi)} \int_{\xi}^{\eta} \beta_{1}(y)\left[e^{\frac{2 i}{\varepsilon}(\phi(y)-\phi(\xi))}-1\right]^{\prime} d y \\
& =-(i \varepsilon)^{2} e^{\frac{2 i}{\varepsilon} \phi(\xi)}\left[\beta_{1}(y) H_{1}\left(\frac{2}{\varepsilon}(\phi(y)-\phi(\xi))\right)\right]_{\xi}^{\eta}+\mathcal{O}_{\varepsilon, h}(\varepsilon h \min (\varepsilon, h)) .
\end{aligned}
$$

$(2.23)$

And this will lead to a consistency error for $Z$ of the order $\mathcal{O}_{\varepsilon, h}\left(\varepsilon^{2} h \min (\varepsilon, h)\right)$. We remark that reversing the order of the two above expansion steps would not yield the desired error order.

With the above ingredients we shall now introduce the first order scheme for solving the reformulated equation (2.6) in the unknown $Z$.

First order scheme. Let $Z_{1}:=Z_{I}$ be the initial condition and let $n=$ $1, \cdots, N-1$.

$$
Z_{n+1}=\left(I+A_{n}^{1}\right) Z_{n}
$$


with the $2 \times 2$ matrix

$$
\begin{aligned}
& A_{n}^{1}:=\varepsilon^{3} \beta_{1}\left(x_{n+1}\right)\left(\begin{array}{cc}
0 & e^{-\frac{2 i}{\varepsilon} \phi\left(x_{n}\right)} H_{1}\left(-\frac{2}{\varepsilon} S_{n}\right) \\
e^{\frac{2 i}{\varepsilon} \phi\left(x_{n}\right)} H_{1}\left(\frac{2}{\varepsilon} S_{n}\right) & 0
\end{array}\right) \\
& -i \varepsilon^{2}\left(\begin{array}{cc}
0 & \beta_{0}\left(x_{n}\right) e^{-\frac{2 i}{\varepsilon} \phi\left(x_{n}\right)}-\beta_{0}\left(x_{n+1}\right) e^{-\frac{2 i}{\varepsilon} \phi\left(x_{n+1}\right)} \\
\beta_{0}\left(x_{n+1}\right) e^{\frac{2 i}{\varepsilon} \phi\left(x_{n+1}\right)}-\beta_{0}\left(x_{n}\right) e^{\frac{2 i}{\varepsilon} \phi\left(x_{n}\right)} & 0
\end{array}\right),
\end{aligned}
$$

and the phase increments

$$
S_{n}:=\phi\left(x_{n+1}\right)-\phi\left(x_{n}\right)=\int_{x_{n}}^{x_{n+1}}\left(\sqrt{a(\tau)}-\varepsilon^{2} \beta(\tau)\right) d \tau .
$$

We remark that finite truncations of the asymptotic expansion (2.19) do not preserve the original skew-symmetry of $m_{1}^{\varepsilon}(\eta ; \xi)$ w.r.t. interchanging $\eta$ and $\xi$. If this property of the approximation is desirable, it is easily recovered by rather extracting the factor $\exp \left(\frac{2 i}{\varepsilon} \phi\left(\frac{\xi+\eta}{2}\right)\right)$ in the first step of (2.18) instead of $\exp \left(\frac{2 i}{\varepsilon} \phi(\xi)\right)$. This will however only intricate the formulae without improving them, such that we shall not pursue this direction in the following.

To construct a second order scheme for (2.15), we truncate (2.16) at $P=2$, which yields

$$
Z\left(x_{n+1}\right)=Z\left(x_{n}\right)+\left[\varepsilon M_{1}^{\varepsilon}\left(x_{n+1} ; x_{n}\right)+\varepsilon^{2} M_{2}^{\varepsilon}\left(x_{n+1} ; x_{n}\right)\right] Z\left(x_{n}\right)+\theta_{n}, \quad \text { with }
$$

$$
\theta_{n}=\mathcal{O}_{\varepsilon, h}\left(\varepsilon^{3} h^{2} \min (\varepsilon, h)\right) .
$$

Proceeding similarly as for the construction of the first order scheme, we shall improve the expansion of $M_{1}^{\varepsilon}\left(x_{n+1} ; x_{n}\right)$ and approximate $M_{2}^{\varepsilon}\left(x_{n+1} ; x_{n}\right)$ in such a manner, that we get a consistency error of the same magnitude as $\theta_{n}$. That means an error for $M_{1}^{\varepsilon}\left(x_{n+1} ; x_{n}\right)$ of the order $\mathcal{O}_{\varepsilon, h}\left(\varepsilon^{2} h^{2} \min (\varepsilon, h)\right)$ and for $M_{2}^{\varepsilon}\left(x_{n+1} ; x_{n}\right)$ of the order $\mathcal{O}_{\varepsilon, h}\left(\varepsilon h^{2} \min (\varepsilon, h)\right)$.

Higher order expansion of $M_{1}^{\varepsilon}$. For the improved expansion of $M_{1}^{\varepsilon}$ we shall perform 2 steps of the standard asymptotic method (2.21) and then 2 steps of the "shifted asymptotic method" (2.18) (in this order!). This yields

$$
\begin{aligned}
m_{1}^{\varepsilon}(\eta ; \xi)= & \left(M_{1}^{\varepsilon}(\eta ; \xi)\right)_{2,1}=\int_{\xi}^{\eta} \beta(y) e^{\frac{2 i}{\varepsilon} \phi(y)} d y \\
= & -i \varepsilon\left[\beta_{0}(y) e^{\frac{2 i}{\varepsilon} \phi(y)}\right]_{\xi}^{\eta}-(i \varepsilon)^{2}\left[\beta_{1}(y) e^{\frac{2 i}{\varepsilon} \phi(y)}\right]_{\xi}^{\eta}+T_{2}^{\varepsilon}(\eta, \xi) . \\
T_{2}^{\varepsilon}(\eta, \xi)= & (i \varepsilon)^{2} \int_{\xi}^{\eta} \beta_{1}^{\prime}(y) e^{\frac{2 i}{\varepsilon} \phi(y)} d y \\
= & -(i \varepsilon)^{3} e^{\frac{2 i}{\varepsilon} \phi(\xi)} \int_{\xi}^{\eta} \beta_{2}(y)\left[e^{\frac{2 i}{\varepsilon}(\phi(y)-\phi(\xi))}-1\right]^{\prime} d y \\
= & -(i \varepsilon)^{3} e^{\frac{2 i}{\varepsilon} \phi(\xi)}\left\{\left[\beta_{2}(y) H_{1}\left(\frac{2}{\varepsilon}(\phi(y)-\phi(\xi))\right)\right]_{\xi}^{\eta}\right. \\
& \left.+i \varepsilon\left[\beta_{3}(y) H_{2}\left(\frac{2}{\varepsilon}(\phi(y)-\phi(\xi))\right)\right]_{\xi}^{\eta}\right\} \\
& +\mathcal{O}_{\varepsilon, h}\left(\varepsilon^{2} h^{2} \min (\varepsilon, h)\right) .
\end{aligned}
$$


Expansion of $M_{2}^{\varepsilon}$. We have

$$
M_{2}^{\varepsilon}(\eta ; \xi)=\int_{\xi}^{\eta} N^{\varepsilon}(y) M_{1}^{\varepsilon}(y ; \xi) d y
$$

such that we shall first use the expansion (2.22), (2.23) of $M_{1}^{\varepsilon}$ to approximate $M_{2}^{\varepsilon}$. Since $N^{\varepsilon}$ and $M_{1}^{\varepsilon}$ are off-diagonal, the $M_{2}^{\varepsilon}$-matrix is diagonal and the entries are conjugate of one another. Thus we will only study the term $m_{2}^{\varepsilon}=\left(M_{2}^{\varepsilon}\right)_{1,1}$.

$$
\begin{aligned}
m_{2}^{\varepsilon}(\eta ; \xi)= & \int_{\xi}^{\eta} N_{1,2}^{\varepsilon}(y)\left(M_{1}^{\varepsilon}\right)_{2,1}(y ; \xi) d y \\
= & \int_{\xi}^{\eta} \beta(y) e^{-\frac{2 i}{\varepsilon} \phi(y)}\left\{(-i \varepsilon)\left[\beta_{0}(x) e^{\frac{2 i}{\varepsilon} \phi(x)}\right]_{\xi}^{y}\right. \\
& \left.-(i \varepsilon)^{2} e^{\frac{2 i}{\varepsilon} \phi(\xi)}\left[\beta_{1}(x) H_{1}\left(\frac{2}{\varepsilon}(\phi(x)-\phi(\xi))\right)\right]_{\xi}^{y}+\mathcal{O}_{\varepsilon, h}(\varepsilon h \min (\varepsilon, h))\right\} d y \\
= & -i \varepsilon \int_{\xi}^{\eta} \beta(y) \beta_{0}(y) d y+i \varepsilon \beta_{0}(\xi) e^{\frac{2 i}{\varepsilon} \phi(\xi)} \int_{\xi}^{\eta} \beta(y) e^{-\frac{2 i}{\varepsilon} \phi(y)} d y \\
& -(i \varepsilon)^{2} e^{\frac{2 i}{\varepsilon} \phi(\xi)} \int_{\xi}^{\eta} \beta(y) \beta_{1}(y) e^{-\frac{2 i}{\varepsilon} \phi(y)} H_{1}\left(\frac{2}{\varepsilon}(\phi(y)-\phi(\xi))\right) d y \\
& +\mathcal{O}_{\varepsilon, h}\left(\varepsilon h^{2} \min (\varepsilon, h)\right) \\
= & I_{1}+I_{2}+I_{3}+\mathcal{O}_{\varepsilon, h}\left(\varepsilon h^{2} \min (\varepsilon, h)\right),
\end{aligned}
$$

where

$$
\begin{aligned}
I_{1}:= & -i \varepsilon \frac{\eta-\xi}{2}\left[\beta(\eta) \beta_{0}(\eta)+\beta(\xi) \beta_{0}(\xi)\right]+\mathcal{O}_{\varepsilon, h}\left(\varepsilon h^{3}\right), \\
I_{2}:= & -\varepsilon^{2} \beta_{0}(\xi)\left[\beta_{0}(\eta) H_{1}\left(\frac{2}{\varepsilon}(\phi(\xi)-\phi(\eta))\right)-i \varepsilon \beta_{1}(\eta) H_{2}\left(\frac{2}{\varepsilon}(\phi(\xi)-\phi(\eta))\right)\right] \\
& +\mathcal{O}_{\varepsilon, h}\left(\varepsilon h^{2} \min (\varepsilon, h)\right), \\
I_{3}:= & {\left[-i \varepsilon^{3} \beta_{0}(y) \beta_{1}(y) H_{2}\left(\frac{2}{\varepsilon}(\phi(\xi)-\phi(y))\right)\right]_{\xi}^{\eta}+\mathcal{O}_{\varepsilon, h}\left(\varepsilon h^{2} \min (\varepsilon, h)\right) . }
\end{aligned}
$$

For $I_{2}$ we used the expansion (2.18) of $m_{1}^{\varepsilon}$. Since the integral $\varepsilon \int_{\xi}^{\eta} \beta(y) \beta_{0}(y) d y$ is not oscillatory, we can approximate $m_{2}^{\varepsilon}$ only up to an error of the order $\mathcal{O}_{\varepsilon, h}\left(\varepsilon h^{3}\right)$. Thus, we cannot attain here the desired order $\mathcal{O}_{\varepsilon, h}\left(\varepsilon h^{2} \min (\varepsilon, h)\right)$. Nevertheless, the resulting scheme will be second order and asymptotically correct for $\varepsilon \rightarrow 0$.

With these ingredients the second order scheme can now be introduced.

Second order scheme. Let $Z_{1}:=Z_{I}$ be the initial condition and let $n=1, \ldots, N-1$.

$$
Z_{n+1}=\left(I+A_{m o d, n}^{1}+A_{n}^{2}\right) Z_{n}
$$


with the matrices

$$
\begin{aligned}
& A_{\text {mod }, n}^{1}:= \\
& -i \varepsilon^{2}\left(\begin{array}{cc}
0 & \beta_{0}\left(x_{n}\right) e^{-\frac{2 i}{\varepsilon} \phi\left(x_{n}\right)}-\beta_{0}\left(x_{n+1}\right) e^{-\frac{2 i}{\varepsilon} \phi\left(x_{n+1}\right)} \\
\beta_{0}\left(x_{n+1}\right) e^{\frac{2 i}{\varepsilon} \phi\left(x_{n+1}\right)}-\beta_{0}\left(x_{n}\right) e^{\frac{2 i}{\varepsilon} \phi\left(x_{n}\right)} & 0
\end{array}\right) \\
& +\varepsilon^{3}\left(\begin{array}{cc}
0 & \beta_{1}\left(x_{n+1}\right) e^{-\frac{2 i}{\varepsilon} \phi\left(x_{n+1}\right)}-\beta_{1}\left(x_{n}\right) e^{-\frac{2 i}{\varepsilon} \phi\left(x_{n}\right)} \\
\beta_{1}\left(x_{n+1}\right) e^{\frac{2 i}{\varepsilon} \phi\left(x_{n+1}\right)}-\beta_{1}\left(x_{n}\right) e^{\frac{2 i}{\varepsilon} \phi\left(x_{n}\right)} & 0
\end{array}\right) \\
& +i \varepsilon^{4} \beta_{2}\left(x_{n+1}\right)\left(\begin{array}{cc}
0 & -e^{-\frac{2 i}{\varepsilon} \phi\left(x_{n}\right)} H_{1}\left(-\frac{2}{\varepsilon} S_{n}\right) \\
e^{\frac{2 i}{\varepsilon} \phi\left(x_{n}\right)} H_{1}\left(\frac{2}{\varepsilon} S_{n}\right) & 0 \\
0 & e^{-\frac{2 i}{\varepsilon} \phi\left(x_{n}\right)} H_{2}\left(-\frac{2}{\varepsilon} S_{n}\right)
\end{array}\right) \\
& -\varepsilon^{5} \beta_{3}\left(x_{n+1}\right)\left(\begin{array}{cc}
e^{\frac{2 i}{\varepsilon} \phi\left(x_{n}\right)} H_{2}\left(\frac{2}{\varepsilon} S_{n}\right) & 0
\end{array}\right)
\end{aligned}
$$

$$
\begin{aligned}
A_{n}^{2}:= & -i \varepsilon^{3}\left(x_{n+1}-x_{n}\right) \frac{\beta\left(x_{n+1}\right) \beta_{0}\left(x_{n+1}\right)+\beta\left(x_{n}\right) \beta_{0}\left(x_{n}\right)}{2}\left(\begin{array}{cc}
1 & 0 \\
0 & -1
\end{array}\right) \\
& -\varepsilon^{4} \beta_{0}\left(x_{n}\right) \beta_{0}\left(x_{n+1}\right)\left(\begin{array}{cc}
H_{1}\left(-\frac{2}{\varepsilon} S_{n}\right) & 0 \\
0 & H_{1}\left(\frac{2}{\varepsilon} S_{n}\right)
\end{array}\right) \\
& +i \varepsilon^{5} \beta_{1}\left(x_{n+1}\right)\left[\beta_{0}\left(x_{n}\right)-\beta_{0}\left(x_{n+1}\right)\right]\left(\begin{array}{cc}
H_{2}\left(-\frac{2}{\varepsilon} S_{n}\right) & 0 \\
0 & -H_{2}\left(\frac{2}{\varepsilon} S_{n}\right)
\end{array}\right) .
\end{aligned}
$$

In this schemes we assumed that the function $\beta$ (which involves the derivatives $a^{\prime}, a^{\prime \prime}$ ) is explicitly "available". Alternatively, $a^{\prime}$ and $a^{\prime \prime}$ could be approximated numerically. In order to compute now the solution of (2.2) and thus get the wave function $\varphi$ as well as its derivative $\varphi^{\prime}$, we have to transform back via

$$
Y_{n}=e^{\frac{i}{\varepsilon} \Phi^{\varepsilon}\left(x_{n}\right)} Z_{n}, \quad U_{n}=P^{-1} Y_{n}, \quad n=1, \ldots, N .
$$

We shall now discuss if these two schemes preserve the current conservation property of problem (2.1). In the continuous case the current density satisfies

$$
\begin{aligned}
j(x) & :=\varepsilon \Im\left(\bar{\varphi}(x) \varphi^{\prime}(x)\right)=-\frac{1}{2 i} U(x)^{\top}\left(\begin{array}{cc}
0 & 1 \\
-1 & 0
\end{array}\right) \bar{U}(x) \\
& =-\frac{1}{2 i} Z(x)^{\top} e^{\frac{i}{\varepsilon} \Phi^{\varepsilon}(x)} P^{-1}\left(\begin{array}{cc}
0 & 1 \\
-1 & 0
\end{array}\right) P e^{-\frac{i}{\varepsilon} \Phi^{\varepsilon}(x)} \bar{Z}(x) \\
& =\frac{1}{2} Z(x)^{\top}\left(\begin{array}{cc}
1 & 0 \\
0 & -1
\end{array}\right) \bar{Z}(x),
\end{aligned}
$$

which is constant along the domain $(0,1)$ due to $(2.6)$. Analogously we have on the discrete level

$$
j_{n}:=\frac{1}{2} Z_{n}^{\top}\left(\begin{array}{cc}
1 & 0 \\
0 & -1
\end{array}\right) \bar{Z}_{n}, \quad 1 \leq n \leq N
$$


As we shall see in Proposition 3.3, the above schemes do not keep the discrete current perfectly constant, but only up to a small error of the order $\mathcal{O}_{\varepsilon, h}\left(\varepsilon^{2}(\min (\varepsilon, h))^{2}\right)$.

In Section 3 we first analyze the convergence of the first order scheme (2.24), (2.25), (2.34) and of the second order scheme (2.31)-(2.34). Then we shall present numerical results obtained with the above schemes, including a check on the (almost) preservation of the current (2.36).

\section{Error analysis and numerical results.}

3.1. Convergence of the method. The next theorem is the main result of this paper. Let us denote in the following by $Z(x)$ (resp. $U(x)$ ) the exact solution of the continuous problem (2.6) (resp. (2.2)). And $\left(Z_{n}\right)_{n=1}^{N}\left(\operatorname{resp} .\left(U_{n}\right)_{n=1}^{N}\right)$ denotes the numerical approximation of $Z\left(x_{n}\right)$ (resp. $U\left(x_{n}\right)$ ), computed via the first order scheme $(2.24),(2.25),(2.34)$ or the second order scheme $(2.31)-(2.34)$ presented in Section 2.2. Then we have the following error estimates:

ThEOREM 3.1. Let Hypothesis A be satisfied. Then the global errors of the first order scheme (2.24), (2.25) satisfies

$$
\begin{gathered}
\left\|Z\left(x_{n}\right)-Z_{n}\right\| \leq C \varepsilon^{2} \min (\varepsilon, h), \quad 1 \leq n \leq N \\
\left\|U\left(x_{n}\right)-U_{n}\right\| \leq C \frac{h^{\gamma}}{\varepsilon}+C \varepsilon^{2} \min (\varepsilon, h), \quad 1 \leq n \leq N .
\end{gathered}
$$

And for the second order scheme (2.31)-(2.33) we have the estimate

$$
\left\|Z\left(x_{n}\right)-Z_{n}\right\| \leq C \varepsilon^{3} h^{2},\left\|U\left(x_{n}\right)-U_{n}\right\| \leq C \frac{h^{\gamma}}{\varepsilon}+C \varepsilon^{3} h^{2}, 1 \leq n \leq N,
$$

with $C$ independent of $n, h$, and $\varepsilon$. Here, $\gamma>0$ is the order of the chosen numerical integration method for computing the phase integral

$$
\Phi^{\varepsilon}(x)=\int_{0}^{x}\left(\sqrt{a(\tau)}-\varepsilon^{2} \beta(\tau)\right) d \tau\left(\begin{array}{cc}
1 & 0 \\
0 & -1
\end{array}\right) .
$$

The discretization step size is $h>0$, and $\|$.$\| denotes any vector norm in \mathbb{C}^{2}$.

Proof. Both schemes can be written under the form

$$
Z_{n+1}=\left(I+B_{n}^{k}\right) Z_{n} ; \quad k=1,2,
$$

where $B_{n}^{1}=A_{n}^{1}$ (first order scheme) and $B_{n}^{2}=A_{\bmod , n}^{1}+A_{n}^{2}$ (second order scheme). With (2.20) we have

(3.6) $A_{n}^{1}=\mathcal{O}_{\varepsilon, h}(\varepsilon \min (\varepsilon, h)), \quad A_{m o d, n}^{1}=\mathcal{O}_{\varepsilon, h}(\varepsilon \min (\varepsilon, h)), \quad A_{n}^{2}=\mathcal{O}_{\varepsilon, h}\left(\varepsilon^{3} h\right)$.

Hence, both schemes are stable, with an $\varepsilon$-independent stability constant.

The consistency error for the first order scheme is given by

$$
(3.7) e_{n}:=\left[\varepsilon M_{1}^{\varepsilon}\left(x_{n+1} ; x_{n}\right)-A_{n}^{1}\right] Z\left(x_{n}\right)+\gamma_{n}, \quad \gamma_{n}:=\sum_{p=2}^{\infty} \varepsilon^{p} M_{p}^{\varepsilon}\left(x_{n+1} ; x_{n}\right) Z\left(x_{n}\right) \text {. }
$$

The order of magnitude of these terms is given by the construction of $A_{n}^{1}$ (cf. (2.23)) and by (2.17):

(3.8) $\varepsilon M_{1}^{\varepsilon}\left(x_{n+1} ; x_{n}\right)-A_{n}^{1}=\mathcal{O}_{\varepsilon, h}\left(\varepsilon^{2} h \min (\varepsilon, h)\right), \quad \gamma_{n}=\mathcal{O}_{\varepsilon, h}\left(\varepsilon^{2} h \min (\varepsilon, h)\right)$. 
This proves the assertion (3.1).

For the second order scheme we have

$$
\begin{aligned}
e_{n} & :=\left[\varepsilon M_{1}^{\varepsilon}\left(x_{n+1} ; x_{n}\right)+\varepsilon^{2} M_{2}^{\varepsilon}\left(x_{n+1} ; x_{n}\right)-B_{n}^{2}\right] Z\left(x_{n}\right)+\theta_{n}, \\
\theta_{n} & :=\sum_{p=3}^{\infty} \varepsilon^{p} M_{p}^{\varepsilon}\left(x_{n+1} ; x_{n}\right) Z\left(x_{n}\right)
\end{aligned}
$$

where $\theta_{n}=\mathcal{O}_{\varepsilon, h}\left(\varepsilon^{3} h^{2} \min (\varepsilon, h)\right)$ due to $(2.17)$. By the construction of $A_{\bmod , n}^{1}$ and $A_{n}^{2}$ we deduce from (2.28) and (2.30):

$$
\begin{aligned}
\varepsilon M_{1}^{\varepsilon}\left(x_{n+1} ; x_{n}\right)+\varepsilon^{2} M_{2}^{\varepsilon}\left(x_{n+1} ; x_{n}\right)-B_{n}^{2} & =\mathcal{O}_{\varepsilon, h}\left(\varepsilon^{3} h^{2} \min (\varepsilon, h)\right)+\mathcal{O}_{\varepsilon, h}\left(\varepsilon^{3} h^{3}\right) \\
& =\mathcal{O}_{\varepsilon, h}\left(\varepsilon^{3} h^{3}\right),
\end{aligned}
$$

implying for the second order scheme

$$
\left\|Z\left(x_{n+1}\right)-Z_{n+1}\right\| \leq C \varepsilon^{3} h^{2}, \quad 1 \leq n \leq N .
$$

To obtain now the initially "desired" solution $U$ of $(2.2)$, we have to transform $Z$ back into $U$ via (2.34). This may introduce an additional error if the phase-matrix (3.4) is computed numerically. Indeed,

$$
\begin{aligned}
U\left(x_{n+1}\right)-U_{n+1}= & P^{-1} e^{\frac{i}{\varepsilon} \Phi^{\varepsilon}\left(x_{n+1}\right)} Z\left(x_{n+1}\right)-P^{-1} e^{\frac{i}{\varepsilon} \Phi_{n+1}^{\varepsilon}} Z_{n+1} \\
= & P^{-1}\left\{\left[e^{\frac{i}{\varepsilon} \Phi^{\varepsilon}\left(x_{n+1}\right)}-e^{\frac{i}{\varepsilon} \Phi_{n+1}^{\varepsilon}}\right] Z\left(x_{n+1}\right)\right. \\
& \left.+e^{\frac{i}{\varepsilon} \Phi_{n+1}^{\varepsilon}}\left[Z\left(x_{n+1}\right)-Z_{n+1}\right]\right\} .
\end{aligned}
$$

Thus we have

$$
\left\|U\left(x_{n+1}\right)-U_{n+1}\right\| \leq C\left\|e^{\frac{i}{\varepsilon} \Phi^{\varepsilon}\left(x_{n+1}\right)}-e^{\frac{i}{\varepsilon} \Phi_{n+1}^{\varepsilon}}\right\|+C\left\|Z\left(x_{n+1}\right)-Z_{n+1}\right\|
$$

The first term on the right hand side of (3.11) is rather unfavorable as it depends on $\frac{1}{\varepsilon}$. Indeed, if we choose an integration method of order $\gamma$ to compute the phase $\Phi^{\varepsilon}$ (for example $\gamma=4$ for the Simpson rule), we will have an error with

$$
\left\|e^{\frac{i}{\varepsilon} \Phi^{\varepsilon}\left(x_{n}\right)}-e^{\frac{i}{\varepsilon} \Phi_{n}^{\varepsilon}}\right\| \leq C \frac{h^{\gamma}}{\varepsilon} .
$$

And this yields the error estimate (3.2) for $U$.

REMARK 3.2. Theorem 3.1 shows that the back-transformation (2.34) from $Z$ to $U$ introduces an unsatisfactory error behaving like $1 / \varepsilon$, due to the numerical approximation of the phase $\Phi^{\varepsilon}$. However, this error term can be avoided in some interesting applications. For example, RTD-models typically involve a piecewise linear potential $a(x)$, for which the phase can be integrated exactly.

Let us now study the discrete current conservation of (2.36).

Proposition 3.3. Under Hypothesis A the numerical schemes introduced in §2.2 conserve the discrete current (2.36), up to an error of

$$
\left|j_{n+1}-j_{n}\right| \leq C \varepsilon^{2}(\min (\varepsilon, h))^{2}, \quad n=1, \ldots, N-1,
$$

which yields $j_{n}=j_{I}+\mathcal{O}_{\varepsilon, h}\left(\varepsilon^{2} \min (\varepsilon, h)\right)$ for each $n=2, \cdots, N$, where $j_{I}:=\varepsilon \Im\left(\bar{\varphi}(0) \varphi^{\prime}(0)\right)$ is the initial current. 
Proof. Using the notations $B_{n}^{1}=A_{n}^{1}$ (first order scheme) and $B_{n}^{2}=A_{\bmod , n}^{1}+A_{n}^{2}$ (second order scheme), we have

$$
\begin{aligned}
j_{n+1}-j_{n} & =\frac{1}{2} Z_{n}^{\top}\left\{\left(I+B_{n}^{k}\right)^{\top}\left(\begin{array}{cc}
1 & 0 \\
0 & -1
\end{array}\right)\left(I+\bar{B}_{n}^{k}\right)-\left(\begin{array}{cc}
1 & 0 \\
0 & -1
\end{array}\right)\right\} \bar{Z}_{n} \\
& =\frac{1}{2} Z_{n}^{\top}\left(B_{n}^{k}\right)^{\top}\left(\begin{array}{cc}
1 & 0 \\
0 & -1
\end{array}\right) \bar{B}_{n}^{k} \bar{Z}_{n} \\
& =-\frac{1}{2}\left[\left(B_{n}^{k}\right)_{12}\left(B_{n}^{k}\right)_{21}-\left(B_{n}^{k}\right)_{11}\left(B_{n}^{k}\right)_{22}\right] Z_{n}^{\top}\left(\begin{array}{cc}
1 & 0 \\
0 & -1
\end{array}\right) \bar{Z}_{n} \\
& =-\frac{1}{2}\left[\left|\left(B_{n}^{k}\right)_{12}\right|^{2}-\left|\left(B_{n}^{k}\right)_{11}\right|^{2}\right] j_{n} .
\end{aligned}
$$

Here we used the above mentioned symmetries of $B_{n}^{k}$, i.e. $\left(\bar{B}_{n}^{k}\right)_{12}=\left(B_{n}^{k}\right)_{21},\left(\bar{B}_{n}^{k}\right)_{11}=$ $\left(B_{n}^{k}\right)_{22}$.

REMARK 3.4. In the first order scheme (where $\left.\left(B_{n}^{1}\right)_{11}=0\right)$ we see from (3.12) that the error of the current has a (negative) sign. Hence, the error does not oscillate around the correct value $j_{I}$, but rather drifts away slowly (see Fig. 3.2). If perfect conservation of the discrete current is required in a specific application, one could easily modify the first order scheme (2.24) as follows:

$$
\tilde{Z}_{n+1}=\left(1-\frac{1}{2}\left|\left(B_{n}^{1}\right)_{12}\right|^{2}\right)^{-1 / 2}\left(I+B_{n}^{1}\right) \tilde{Z}_{n}
$$

And the resulting first order scheme still satisfies the convergence estimate of Theorem 3.1.

3.2. Numerical results. We shall present now the numerical results obtained with the two schemes introduced in Section 2.2. For our numerical tests we chose $a(x)=(x+1 / 2)^{2}$, such that the phase

$$
\Phi^{\varepsilon}(x)=\int_{0}^{x}\left(\sqrt{a(\tau)}-\varepsilon^{2} \beta(\tau)\right) d \tau\left(\begin{array}{cc}
1 & 0 \\
0 & -1
\end{array}\right),
$$

is explicitly computable. Hence, the $h^{\gamma} / \varepsilon$-term in (3.2) drops and the scheme is asymptotically correct w.r.t. $\varepsilon$ in this example. Thus, even for $h=1$ fixed, the error decays like $\mathcal{O}\left(\varepsilon^{3}\right) \quad$ (cf. Fig. 3.1 and Theorem 3.1). This remarkable behavior is not shared by most schemes from the literature (recall the discussion on the asymptotic limit of (2.6) in $\S 2.1)$.

Figure 3.1 shows the numerical error of the first order scheme $(2.24),(2.25)$ and the second order scheme $(2.31)-(2.33)$, as a function of the discretization step $h$, and for various $\varepsilon$-values. Plotted are the errors in the $L^{2}(0,1)$-norm between the numerical solution and a reference solution, which is computed with the same method on a very fine grid. As can be observed, the plots seem to conform to the error estimates of Theorem 3.1. Indeed, one can read from the plots an error of the order $\mathcal{O}_{\varepsilon, h}\left(\varepsilon^{2} \min (h, \varepsilon)\right)$ for the first order scheme and of the order $\mathcal{O}_{\varepsilon, h}\left(\varepsilon^{3} h^{2}\right)$ for the second order scheme. We remark that the relevant regime is here $h<0.1$ since, otherwise, the given potential $a(x)$ would not be resolved on the grid. Note that, for a significant range of $h$ - and $\varepsilon$-values, these error curves lie well below the "standard" computer precision (in our case $10^{-16}$ for double precision). In order to exclude round-off errors here, these computations were carried out in quadruple precision. The error curves 

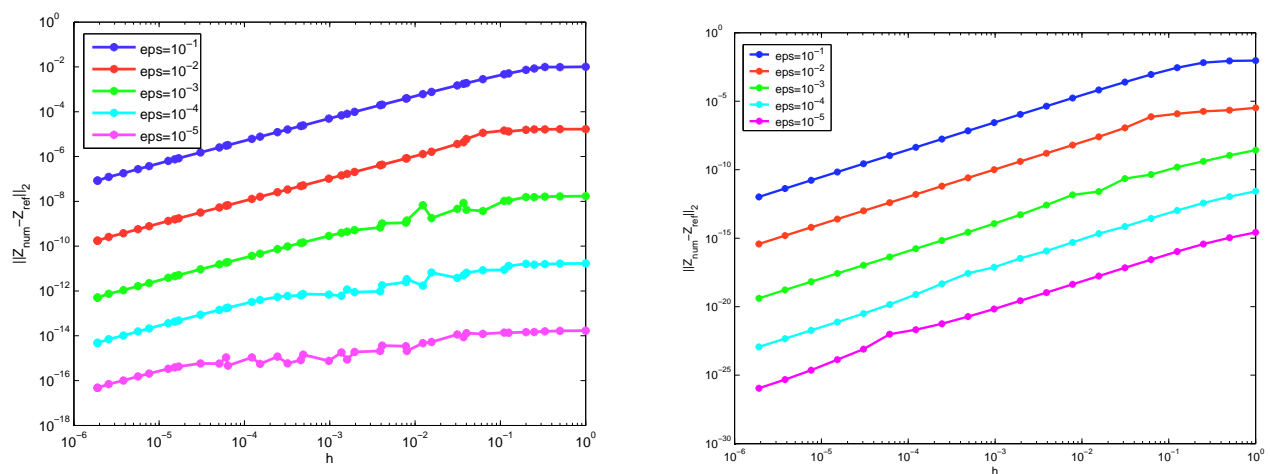

FIG. 3.1. Absolute error (in the $L^{2}(0,1)-$ norm and $\log -\log$ scale) between the computed solution $Z_{\text {num }}$ and a reference solution $Z_{\text {ref }}$ as a function of $h$ and for several $\varepsilon$-values. Left: first order scheme. Right: second order scheme.

in (Fig. 3.1 left) are not monotone, but show a slight resonance behavior at distinct values of $h$. This behavior has already been reported for similar methods (cf. [11]). In $\S 3.3$ we shall give a detailed analysis of this phenomenon, showing that it is not a significant problem.

In Figure 3.2 we plotted the current drift $j(x)-j_{I}$ for the first order scheme. It shows that the current is almost conserved (as a function of $x$ ) by the scheme.
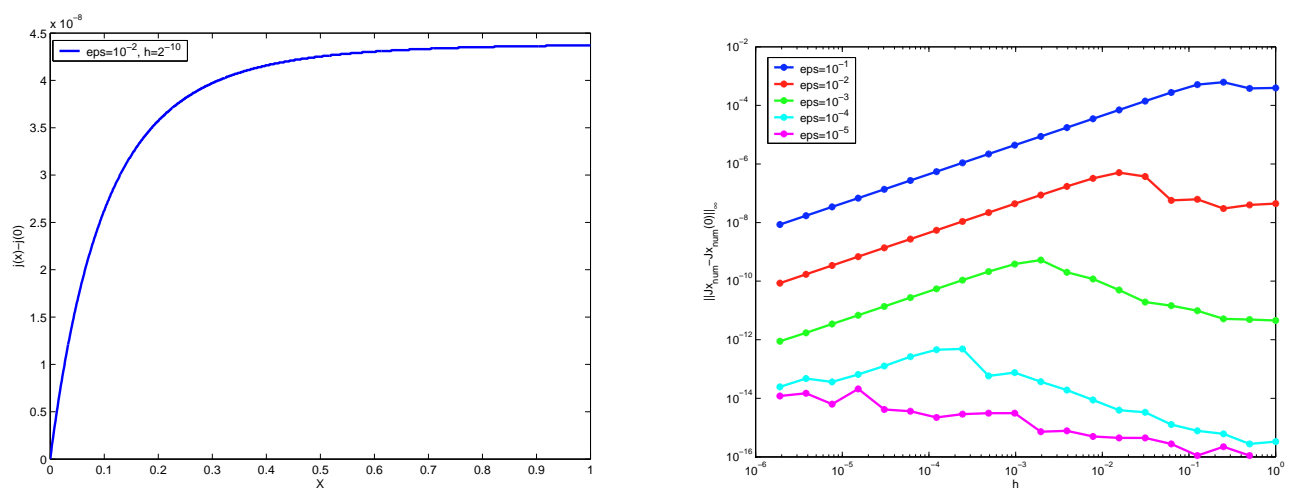

FIG. 3.2. Approximate conservation of the current by the first order scheme. Left: $j(x)-j_{I}$ for one fixed $h$ and $\varepsilon$. Right: Error in current, $\left\|j(.)-j_{I}\right\|_{\infty}$ for different step sizes $h$ and different $\varepsilon$-values.

3.3. Refined error analysis for a simplified first order scheme. The goal of this section is twofold: We shall present a modified first order scheme for (2.15) which is simpler than (2.24), (2.25), but also slightly less accurate: cp. Fig. 3.1 (left) and Fig. 3.3. Moreover we shall sketch a refined error analysis for numerical schemes like those of $\S 2.2$. For the convergence rates obtained in $\S 3.1$ we just considered the consistency error of each numerical step. But for oscillatory problems this may yield over-conservative error estimates. For such problems the true error accumulation, however, may exhibit some cancellation phenomena as long as the grid step stays away from certain "resonant values". Here, we shall illustrate this phenomenon for the simplified first order scheme, where the approximation of $M_{1}^{\varepsilon}$ (in Step 2 of §2.2) is of lower order than the series truncation in Step 1 of $\S 2.2$. Making just one step 
of the shifted asymptotic method in (2.18) yields the following "modified first order scheme". Let $Z_{1}:=Z_{I}$ be the initial condition and let $n=1, \cdots, N-1$.

\section{Modified first order scheme.}

$$
Z_{n+1}=Z_{n}+\tilde{A}_{n}^{1} Z_{n}
$$

with the $2 \times 2$ matrix

$$
\begin{aligned}
\tilde{A}_{n}^{1} & :=-i \varepsilon^{2} \beta_{0}\left(x_{n+1}\right)\left(\begin{array}{cc}
0 & e^{-\frac{2 i}{\varepsilon} \phi\left(x_{n}\right)}-e^{-\frac{2 i}{\varepsilon} \phi\left(x_{n+1}\right)} \\
e^{\frac{2 i}{\varepsilon} \phi\left(x_{n+1}\right)}-e^{\frac{2 i}{\varepsilon} \phi\left(x_{n}\right)} & 0
\end{array}\right) \\
& =-i \varepsilon^{2} \beta_{0}\left(x_{n+1}\right)\left(\begin{array}{cc}
0 & -e^{-\frac{2 i}{\varepsilon} \phi\left(x_{n}\right)} H_{1}\left(-\frac{2}{\varepsilon} S_{n}\right) \\
e^{\frac{2 i}{\varepsilon} \phi\left(x_{n}\right)} H_{1}\left(\frac{2}{\varepsilon} S_{n}\right) & 0
\end{array}\right),
\end{aligned}
$$

and the notations of Section 2.2. In analogy to $\S 3.1$ this scheme satisfies the following result:

THEOREM 3.5. Let Hypothesis A be satisfied. Then the global errors of the modified first order scheme satisfy

$$
\begin{aligned}
& \left\|Z\left(x_{n}\right)-Z_{n}\right\|_{2} \leq C \varepsilon \min (\varepsilon, h), \\
& \left\|U\left(x_{n}\right)-U_{n}\right\|_{2} \leq C \frac{h^{\gamma}}{\varepsilon}+C \varepsilon \min (\varepsilon, h), \quad 1 \leq n \leq N,
\end{aligned}
$$

with $C$ independent of $h$ and $\varepsilon$. Here, $\gamma>0$ is again the order of the chosen numerical integration method for computating of the phase integral

$$
\Phi^{\varepsilon}(x)=\int_{0}^{x}\left(\sqrt{a(\tau)}-\varepsilon^{2} \beta(\tau)\right) d \tau\left(\begin{array}{cc}
1 & 0 \\
0 & -1
\end{array}\right) .
$$

The discretization step size is $h>0$.

The difference between the first order scheme of Section 2.2 and the modified first order scheme is the $\varepsilon$-power in the error estimate. Now we continue with the numerical example from $\S 3.2$. Figure 3.3 shows the $L^{2}$-errors between the computed solution $Z_{\text {num }}$ via (3.13)-(3.14) and a (more accurate) reference solution $Z_{\text {ref }}$. Note that the $\varepsilon$-asymptotics of the error in Figure 3.3 is actually much better (i.e. smaller) than estimated in Theorem 3.5. Indeed, one can read from the plots an error of the order $\mathcal{O}_{\varepsilon, h}\left(\varepsilon^{2} \min (h, 1)\right)$ rather than the expected $\mathcal{O}_{\varepsilon, h}(\varepsilon \min (\varepsilon, h))$. To understand this phenomenon we shall analyze in much more detail the error estimate obtained in Theorem 3.5. Anticipating that analysis, we mention that the improved error behavior is due to cancellation effects in successive integration steps - at least for "most" values of $h$. However, for certain resonant $h$-values (e.g. $h=2^{-11}, \varepsilon=10^{-4}$ or $h=2^{-14}, \varepsilon=10^{-5}$ ) the error is as predicted by Theorem 3.5. Hence, this theorem seems to be sharp in general, and the smaller-than-expected error makes the simplified scheme competitive with the first order scheme of $\S 2.2$.

Let us now improve the error analysis for the modified first order scheme (3.13)(3.14) in order to explain the phenomenon detected in Figure 3.3. With the notations of Section 3.1 (particularly from the proof of Theorem 3.1) and the usual error propagation in one-step ODE-schemes we obtain

$$
\left\|Z\left(x_{n+1}\right)-Z_{n+1}\right\| \leq\left\|\sum_{j=1}^{n}\left[\Pi_{l=j+1}^{n}\left(I+\tilde{A}_{l}^{1}\right)\right] e_{j}\right\|, \quad 1 \leq n<N
$$




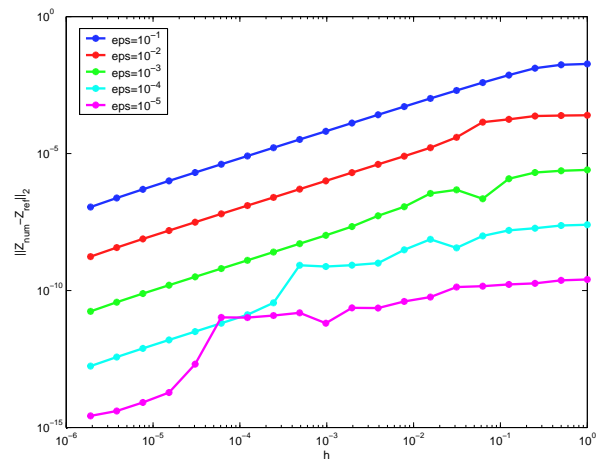

FIG. 3.3. Absolute error between the computed solution $Z_{\text {num }}$ and a reference solution $Z_{\text {ref }}$ as a function of $h$, for the modified first order scheme.

where $e_{j}:=\left[\varepsilon M_{1}^{\varepsilon}\left(x_{j+1} ; x_{j}\right)-\tilde{A}_{j}^{1}\right] Z\left(x_{j}\right)+\gamma_{j}$ is the consistency error. From (2.17) we have

$$
M_{1}^{\varepsilon}\left(x_{n+1} ; x_{n}\right)-\tilde{A}_{n}^{1}=\mathcal{O}_{\varepsilon, h}(\varepsilon h \min (\varepsilon, h)), \quad \gamma_{j}:=\mathcal{O}_{\varepsilon, h}\left(\varepsilon^{2} h \min (\varepsilon, h)\right) .
$$

In particular we are interested in a refined estimate of the term

$$
T_{n}:=\left\|\sum_{j=1}^{n}\left[\Pi_{l=j+1}^{n}\left(I+\tilde{A}_{l}^{1}\right)\right]\left(\varepsilon M_{1}^{\varepsilon}\left(x_{j+1} ; x_{j}\right)-\tilde{A}_{j}^{1}\right) Z\left(x_{j}\right)\right\|, \quad 1 \leq n<N,
$$

since $\sum_{j=1}^{n}\left[\Pi_{l=j+1}^{n}\left(I+\tilde{A}_{l}^{1}\right)\right] \gamma_{j}$ is already of the order $\mathcal{O}_{\varepsilon, h}\left(\varepsilon^{2} \min (\varepsilon, h)\right)$. Due to the fact that $\tilde{A}_{l}^{1}=\mathcal{O}_{\varepsilon, h}(\varepsilon \min (\varepsilon, h))$, we can show that $\Pi_{l=j+1}^{n}\left(I+\tilde{A}_{l}^{1}\right)=I+$ $\mathcal{O}_{\varepsilon, h}(N \varepsilon \min (\varepsilon, h))=I+\mathcal{O}_{\varepsilon, h}(\varepsilon)$, where $N=1+1 / h$ is the number of grid points. With (3.17) this yields

$$
T_{n} \leq\left\|\sum_{j=1}^{n}\left(\varepsilon M_{1}^{\varepsilon}\left(x_{j+1} ; x_{j}\right)-\tilde{A}_{j}^{1}\right) Z\left(x_{j}\right)\right\|+\mathcal{O}_{\varepsilon, h}\left(\varepsilon^{2} \min (\varepsilon, h)\right) .
$$

As $Z(x)=Z_{I}+\mathcal{O}\left(\varepsilon^{2}\right)$, we have

$$
\left\|\sum_{j=1}^{n}\left(\varepsilon M_{1}^{\varepsilon}\left(x_{j+1} ; x_{j}\right)-\tilde{A}_{j}^{1}\right) Z\left(x_{j}\right)\right\| \leq C\left\|\sum_{j=1}^{n}\left(\varepsilon M_{1}^{\varepsilon}\left(x_{j+1} ; x_{j}\right)-\tilde{A}_{j}^{1}\right)\right\|+\mathcal{O}_{\varepsilon, h}\left(\varepsilon^{3} \min (\varepsilon, h)\right) .
$$

Thus, the improvement visible in Figure 3.3 can only come from some cancellation in the term $E_{n}:=\sum_{j=1}^{n}\left(\varepsilon M_{1}^{\varepsilon}\left(x_{j+1} ; x_{j}\right)-\tilde{A}_{j}^{1}\right)$. Let us analyze this term in more detail:

$$
\begin{aligned}
\left(E_{n}\right)_{21} & =\sum_{j=1}^{n}\left(\varepsilon M_{1}^{\varepsilon}\left(x_{j+1} ; x_{j}\right)-\tilde{A}_{j}^{1}\right)_{21} \\
& =i \varepsilon^{2} \sum_{j=1}^{n} e^{\frac{2 i}{\varepsilon} \phi\left(x_{j}\right)} \int_{x_{j}}^{x_{j+1}} \beta_{0}^{\prime}(y) H_{1}\left(\frac{2}{\varepsilon}\left(\phi(y)-\phi\left(x_{j}\right)\right)\right) d y .
\end{aligned}
$$

A rough estimate will lead at this step to $E_{n}=\mathcal{O}_{\varepsilon, h}(\varepsilon \min (\varepsilon, h))$, which is exactly 
what we got in Theorem 3.5. But continuing by partial integration leads to

$$
\begin{aligned}
& \left(E_{n}\right)_{21}=-\varepsilon^{3} \sum_{j=1}^{n} e^{\frac{2 i}{\varepsilon} \phi\left(x_{j}\right)} \int_{x_{j}}^{x_{j+1}} \beta_{1}(y) H_{2}\left(\frac{2}{\varepsilon}\left(\phi(y)-\phi\left(x_{j}\right)\right)\right)^{\prime} d y \\
& =\varepsilon^{3} \sum_{j=1}^{n} e^{\frac{2 i}{\varepsilon} \phi\left(x_{j}\right)}\left\{\int_{x_{j}}^{x_{j+1}} \beta_{1}^{\prime}(y) H_{2}\left(\frac{2}{\varepsilon}\left(\phi(y)-\phi\left(x_{j}\right)\right)\right) d y-\beta_{1}\left(x_{j+1}\right) H_{2}\left(\frac{2}{\varepsilon} S_{j}\right)\right\} \\
& =\mathcal{O}_{\varepsilon, h}(\varepsilon h \min (\varepsilon, h))-\varepsilon^{3} \sum_{j=1}^{n} e^{\frac{2 i}{\varepsilon} \phi\left(x_{j}\right)} \beta_{1}\left(x_{j+1}\right) H_{2}\left(\frac{2}{\varepsilon} S_{j}\right) \\
& =\mathcal{O}_{\varepsilon, h}(\varepsilon h \min (\varepsilon, h))-\varepsilon h \min (\varepsilon, h) \sum_{j=1}^{n} c_{j} e^{\frac{2 i}{\varepsilon} \phi\left(x_{j}\right)}
\end{aligned}
$$

with some constants $c_{j}>0$ bounded independently of $N$ and $\varepsilon$. Thus, the behavior of $\left(E_{n}\right)_{21}$ depends mostly on the term

$$
\tau(n ; \varepsilon, h):=\sum_{j=1}^{n} c_{j} e^{\frac{2 i}{\varepsilon} \phi\left(x_{j}\right)}, \quad 1 \leq n<N
$$

A cancellation in this term would explain the reduced numerical error as plotted in Figure 3.3. In the worst case we would have $\tau=\mathcal{O}_{\varepsilon, h}(1 / h)$, which occurs in the example $c_{j}=C$ and $\phi\left(x_{j}\right)=k_{j} \pi \varepsilon$ with some $k_{j} \in \mathbb{Z}$. Then, (3.18), (3.19) would lead to an error of

$$
\begin{aligned}
\left\|Z\left(x_{n+1}\right)-Z_{n+1}\right\| & =\mathcal{O}_{\varepsilon, h}\left(\varepsilon^{2} \min (\varepsilon, h)\right)+\mathcal{O}_{\varepsilon, h}(\varepsilon h \min (\varepsilon, h))+\mathcal{O}_{\varepsilon, h}(\varepsilon \min (\varepsilon, h)) \\
& =\mathcal{O}_{\varepsilon, h}(\varepsilon \min (\varepsilon, h))
\end{aligned}
$$

which was asserted in Theorem 3.5. The situation at hand is reminiscent of "Method 2 " in [12]. There, the dominant error term is also multiplied by an oscillatory factor. And this leads to a reduced error order at resonant values of the spatial grid.

To illustrate the possible behavior of $\tau$, we shall now analyze three examples. First we take $c_{j}=C$ and the linear phase function $\phi\left(x_{j}\right):=x_{j}=(j-1) h$ for $j=1, \cdots, N$. Hence

$$
\tau=C \sum_{j=1}^{n}\left(e^{\frac{2 i}{\varepsilon} h}\right)^{j-1}=C \frac{1-e^{\frac{2 i}{\varepsilon} h n}}{1-e^{\frac{2 i}{\varepsilon} h}} .
$$

Thus, $\tau$ is uniformly bounded (in $n, h, \varepsilon$ ), if $h / \varepsilon$ stays away from $k \pi, k \in \mathbb{N}_{0}$. Using $\tau=\mathcal{O}_{\varepsilon, h}(1)$ in (3.18), (3.19) then yields an over all error of the order $\mathcal{O}_{\varepsilon, h}\left(\varepsilon^{2} \min (\varepsilon, h)\right)$ $+\mathcal{O}_{\varepsilon, h}(\varepsilon h \min (\varepsilon, h))=\mathcal{O}_{\varepsilon, h}\left(\varepsilon^{2} h\right)$, which is exactly what we see in Figure 3.3 (for $h<0.1$ and for a different $\phi$, though). However, for resonant grid steps (i.e. $\frac{h}{\pi \varepsilon} \in \mathbb{N}_{0}$ ) we have $\tau=C n=\mathcal{O}_{\varepsilon, h}(1 / h)$ (cf. Figure 3.4).

As a second example we consider $c_{j}=C$ and the quadratic phase function $\phi\left(x_{j}\right):=x_{j}^{2}=(j-1)^{2} h^{2} ; j=1, \cdots, N$, which is related to our test example in $\S 3.2$. This yields

$$
\tau=C \sum_{j=1}^{n} e^{\frac{2 i}{\varepsilon} h^{2}(j-1)^{2}}=C \sum_{j=1}^{n} e^{i \pi \sigma(j-1)^{2}}, \quad 1 \leq n<N
$$



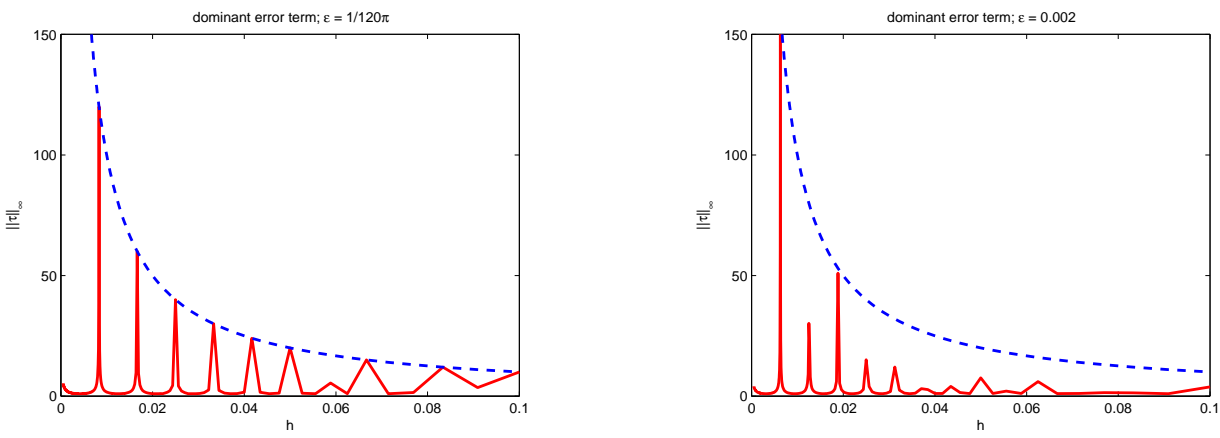

FIG. 3.4. Solid line: Oscillatory sum $\max _{1 \leq n<N}|\tau(n)|$ as a function of $h=1 / N, N \in \mathbb{N}$ for a linear phase function and two values of $\varepsilon$. Left: $\varepsilon=\frac{1}{120 \pi}$ implies that $h / \varepsilon$ hits exactly the resonance values $\pi, 2 \pi, \ldots, 6 \pi$; right: For $\varepsilon=0.002$ the quotient $h / \varepsilon$ is only close to resonances. Dashed line: $1 / h$ is the upper limit of $\|\tau\|_{\infty}$.

where $\sigma=\frac{2}{\pi \varepsilon} h^{2}$. Hardy and Littlewood [6] studied this type of series: for irrational $\sigma$ fixed, we have

$$
\sum_{j=1}^{n} e^{i \pi \sigma(j-1)^{2}}=o(n) \quad \text { as } n \rightarrow \infty
$$

But for rational $\sigma$ we only have $\tau=\mathcal{O}(n)$ as $n \rightarrow \infty$. In Th. III of [23] this result was refined, showing that $\tau=\mathcal{O}\left(\sqrt{n}(\log n)^{\frac{1}{4}+\epsilon}\right)$ for almost all $\sigma \in \mathbb{R}$. And this illustrates the delicate resonance behavior of oscillatory sums like (3.20).
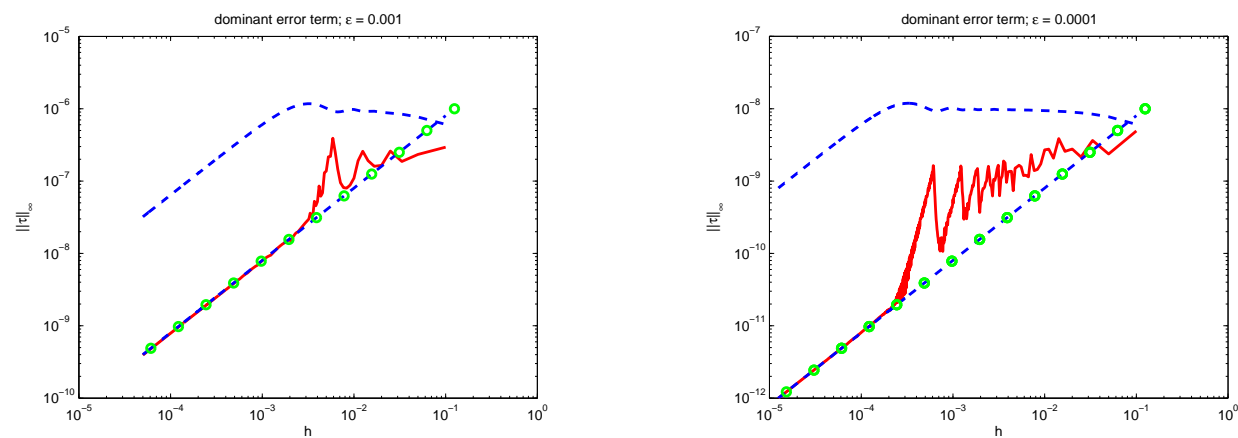

FIG. 3.5. Solid line: Oscillatory sum $\|\tau\|_{\infty}$ as a function of $h=1 / N, N \in \mathbb{N}$ for our numerical example and $\varepsilon=10^{-3}, 10^{-4}$. The lower dashed line is the function $8 \varepsilon^{2} h$ for the asymptotic behavior of $\|\tau\|_{\infty}$ for $h$ small. The circles indicate the positions of the discretization steps chosen in Fig. 3.3. The upper dashed line reflects very closely the error estimate of order $\mathcal{O}_{\varepsilon, h}(\varepsilon \min (\varepsilon, h))$ given in Theorem 3.5.

Finally, we compute $\tau$ for the modified first order scheme applied to the numerical example of $\S 3.2$, i.e. with the coefficients $c_{j}$ given by (3.19). Figure 3.5 shows $\|\tau\|_{\infty}$ as a function of $h=1 / N, N \in \mathbb{N}$. As a comparison we also include the function $C \varepsilon^{2} h$ with the fitted constant $C=8$, which represents very well the asymptotic behavior of $\|\tau\|_{\infty}$ for $h$ small. Observe that $h=2^{-8}$ (for $\varepsilon=10^{-3}$, left picture) and $h=2^{-11}$ (for $\varepsilon=10^{-4}$, right picture) are close to resonances of $\tau$. And this is clearly reflected by the increased simulation error at these precise values of $h$ (cf. Figure 3.3). The 
included upper bound is obtained by computing $\sum_{j=1}^{n}\left|c_{j}\right|$ in (3.19), and this corresponds precisely to the error estimate of order $\mathcal{O}_{\varepsilon, h}(\varepsilon \min (\varepsilon, h))$ given in Theorem 3.5.

To sum up, the estimates of Theorem 3.5 are (in general) sharp, in the sense of guaranteeing first order convergence (independently of resonances - a property also shared by the methods in [11]). But "most of the time" we obtain even better simulation results, as seen in Figure 3.3. Finally, we remark that also Fig. 3.1 shows some (quite mild) resonance behavior at certain values of $h$. But we shall not extend here the refined error analysis to those schemes.

4. Conclusion. We have introduced in this paper a new method for solving highly oscillating differential equations. This method differs from standard numerical methods by using first an analytic reformulation of the problem in order to eliminate the high oscillations of the solution. The transformed "smooth" (i.e. less oscillatory) equation was then numerically discretized. Error estimates underlined the advantages of the new method: For phase integrals that can be computed analytically, the method is even asymptotically correct w.r.t. the small problem parameter $\varepsilon$. In this case the error decreases as $\varepsilon \rightarrow 0$, even for a fixed spatial grid size $h$.

Acknowledgments. The authors acknowledge support by the project No. BLAN07-2 212988 entitled "QUATRAIN", funded by the Agence Nationale de la Recherche and by the "Wissenschaftskolleg Differentialgleichungen" of the FWF. The authors would like to thank TU-Wien, Université Paul Sabatier de Toulouse, and Université de Provence for its support. Fruitful discussions with Jens Geier and Markus Melenk, as well as many clarifying suggestings by the anonymous referees are acknowledged.

\section{REFERENCES}

[1] A. Arnold, Mathematical Properties of Quantum Evolution Equations, in Quantum Transport - Modelling, Analysis and Asymptotics, Lecture Notes in Mathematics 1946, G. Allaire, A. Arnold, P. Degond, Th.Y. Hou, Springer, Berlin, 2008.

[2] N. Ben Abdallah, P. Degond, P. A. Markowich, On a one-dimensional Schrödinger-Poisson scattering model, ZAMP 48 (1997), pp. 35-55.

[3] N. Ben Abdallah, O. Pinaud, Multiscale simulation of transport in an open quantum system: Resonances and WKB interpolation, J. Comput. Phys., 213 (2006), no. 1, pp. 288-310.

[4] P. Degond, S. Gallego, F. Méhats, An asymptotic preserving scheme for the Schrödinger equation in the semiclassical limit, C.R. Acad. Sci. Paris, Ser. I, 345 (2007), no. 9, pp. 531-536.

[5] E. Hairer, C. Lubich, G. Wanner, Geometric Numerical Integration: Structure-Preserving Algorithms for Ordinary Differential Equations, 2nd Ed., Springer-Verlag, Berlin Heidelberg, 2006.

[6] G. H. Hardy, J. E. Littlewood, Some problems of diophantine approximation, Acta Mathematica, 37 (1914), pp. 155-191.

[7] F. Ihlenburg, I. Babuška, Finite element solution of the Helmholtz equation with high wave number. I. The h-version of the FEM, Comput. Math. Appl., 30 (1995), no. 9, pp. 9-37.

[8] F. Ihlenburg, I. Babuška, Finite element solution of the Helmholtz equation with high wave number. II. The h-p version of the FEM, SIAM J. Numer. Anal., 34 (1997), no. 1, pp. $315-358$.

[9] A. Iserles, On the Global Error of Discretization Methods for Highly-Oscillatory Ordinary Differential Equations, BIT, 42 (2002), no. 3, pp. 561-599.

[10] A. Iserles, S.P. Nørsett, S. Olver, Highly oscillatory quadrature: The story so far, in A. Bermudez de Castro, ed., Proceeding of ENuMath, Santiago de Compostella (2006), Springer Verlag, 2006, pp. 97-118.

[11] T. Jahnke, Long-time-step integrators for almost-adiabatic quantum dynamics, SIAM J. Sci. Comp., 25 (2004), pp. 2145-2164. 
[12] T. Jahnke, C. Lubich, Numerical integrators for quantum dynamics close to the adiabatic limit, Numerische Mathematik, 94 (2003), pp. 289-314.

[13] S. Jin, L. Pareschi, G. Toscani, Diffusive relaxation schemes for multiscale discrete-velocity kinetic equations, SIAM J. Numer. Anal., 35 (1998), no. 6, pp. 2405-2439.

[14] A. Klar, L. Pareschi, M. Seaid, Uniformly accurate schemes for relaxation approximations to fluid dynamic equations, Appl. Math. Lett., 16 (2003), no. 7, pp. 1123-1127.

[15] L.D. Landau, E.M. Lifschitz, Quantenmechanik, Akademie-Verlag, Berlin, 1985.

[16] C. S. Lent, D. J. Kirkner, The Quantum Transmitting Boundary Method, J. Appl. Phys., 67 (1990), pp. 6353-6359.

[17] K. Lorenz, T. Jahnke, C. Lubich, Adiabatic integrators for highly oscillatory second-order linear differential equations with time-varying eigendecomposition, BIT, 45 (2005), no. 1, pp. 91115 .

[18] J.M. Melenk, On the convergence of Filon quadrature, J. Comput. Appl. Math., 234 (2010), no. 6, pp. 1692-1701.

[19] C. Negulescu, Numerical analysis of a multiscale finite element scheme for the resolution of the stationary Schroedinger equation, Numerische Mathematik, 108 (2008), no. 4, pp. 625-652.

[20] C. Negulescu, N. Ben Abdallah, M. Mouis, An accelerated algorithm for 2D simulations of the quantum ballistic transport in nanoscale MOSFETs, Journal of Computational Physics, 225 (2007), no. 1, pp. 74-99.

[21] S. Olver, Moment-free numerical integration of highly oscillatory functions, IMA J. Numer. Analy., 26 (2006), pp. 213-227.

[22] S. Olver, Moment-free numerical approximation of highly oscillatory integrals with stationary points, European J. Appl. Math., 18 (2007), no. 4, pp. 435-447.

[23] Z. Zalcwasser, Sur les polynomes associés aux fonctions modulaires $\vartheta$, Studia Math., 7 (1938), pp. $16-35$. 